\title{
Neuroprotective Effects of VEGF-A Nanofiber Membrane and FAAH Inhibitor URB597 Against Oxygen-Glucose Deprivation-Induced Ischemic Neuronal Injury
}

\author{
Da-Peng Wang' \\ Kai-Yan Jin' \\ Peng Zhao ${ }^{2}$ \\ Qi $\operatorname{Lin}^{3}$ \\ Kai Kang ${ }^{4}$ \\ Jian $\mathrm{Hai}^{1}$
}

'Department of Neurosurgery, Tong Ji Hospital, Tong Ji University School of Medicine, Shanghai, 200065, People's Republic of China; ${ }^{2}$ Institute for Translational Medicine, Institute for Biomedical Engineering and Nanoscience, Shanghai East Hospital, Tongji University School of Medicine, Shanghai, 200092, People's Republic of China; ${ }^{3}$ Department of Pharmacy, Institutes of Medical Sciences, Shanghai Jiao Tong University School of Medicine, Shanghai, 200025, People's Republic of China; ${ }^{4}$ Department of Research and Surveillance Evaluation, Shanghai Center for Health Promotion, Shanghai, 200040, People's Republic of China
Correspondence: Da-Peng Wang; Jian Hai Department of Neurosurgery, Tong Ji Hospital, Tong Ji University School of Medicine, No. 389, Xincun Road, Putuo District, Shanghai, 200065, People's Republic of China

Fax +86-2I-66III 093

Email wdpboj@126.com;

haijiandoct@zoho.com.cn
Introduction: Brain ischemia is a common neurological disorder worldwide that activates a cascade of pathophysiological events involving decreases in oxygen and glucose levels. Despite substantial efforts to explore its pathogenesis, the management of ischemic neuronal injury remains an enormous challenge. Accumulating evidence suggests that VEGF modified nanofiber (NF) materials and the fatty-acid amide hydrolase (FAAH) inhibitor URB597 exert an influence on alleviating ischemic brain damage. We aimed to further investigate their effects on primary hippocampal neurons, as well as the underlying mechanisms following oxygen-glucose deprivation (OGD).

Methods: Different layers of VEGF-A loaded polycaprolactone (PCL) nanofibrous membranes were first synthesized by using layer-by-layer (LBL) self-assembly of electrospinning methods. The physicochemical and biological properties of VEGF-A NF membranes, and their morphology, hydrophilicity, and controlled-release of VEGF-A were then estimated. Furthermore, the effects of VEGF-A NF and URB597 on OGD-induced mitochondrial oxidative stress, inflammatory responses, neuronal apoptosis, and endocannabinoid signaling components were assessed.

Results: The VEGF-A NF membrane and URB597 can not only promote hippocampal neuron adhesion and viability following OGD but also exhibited antioxidant/anti-inflammatory and mitochondrial membrane potential protection. The VEGF-A NF membrane and URB597 also inhibited OGD-induced cellular apoptosis through activating CB1R signaling. These results indicate that VEGF-A could be controlled-released by LBL self-assembled NF membranes.

Discussion: The VEGF-A NF membrane and URB597 displayed positive synergistic neuroprotective effects through the inhibition of mitochondrial oxidative stress and activation of CB1R/PI3K/AKT/BDNF signaling, suggesting that a VEGF-A loaded NF membrane and the FAAH inhibitor URB597 could be of therapeutic value in ischemic cerebrovascular diseases.

Keywords: brain ischemia, cannabinoid receptor, fatty-acid amide hydrolase, mitochondrial oxidative stress, nanofiber, VEGF-A

\section{Introduction}

Brain ischemia, a basic hemodynamic characteristic of ischemic cerebrovascular diseases, is commonly found in the elderly and is highly prevalent among patients with hypertension, hyperlipidemia, and cerebrovascular abnormalities. It is a crucial risk 
factor for the development of transient ischemic attack, cognitive decline, and ischemic and hemorrhagic strokes. ${ }^{1}$ Annually, 15 million people worldwide suffer a stroke. Ischemic stroke, accounting for $80 \%$ of stroke, is becoming the leading cause of death and long-term disability in Western societies. ${ }^{2}$ Stroke causes insufficient blood flow to the brain tissue, which in turn leads to oxygen-glucose deprivation (OGD). ${ }^{3}$ Nerve cell viability critically depends on blood delivery of oxygen and nutrients. OGD can result in cellular energy and homeostasis failure, evoking various pathophysiological processes, such as inflammatory responses, oxidative stress, neuronal loss, and apoptosis. ${ }^{4}$ However, there are currently no effective therapeutic targets for the treatment of this ischemic neuronal injury.

Nanofiber (NF) materials have been extensively studied and developed because they embrace considerable promise for broad application and offer the excellent features of nanostructured materials. ${ }^{5} \mathrm{NFs}$ show a larger number of general and technical features, such as the high surface-area-to-volume ratio, porosity, tunability, and biocompatibility. ${ }^{6}$ These remarkable qualities make them ideal candidates for a wide range of biomedical and healthcare applications, including cell or protein attachment, wound healing, drug delivery, tumor detection, tissue engineering, and regenerative medicine. ${ }^{6}$ Recently, a number of studies have focused on NF materials that offer promising prospects for the treatment of various neurological diseases, such as spinal cord injury, subarachnoid hemorrhage, and stroke. ${ }^{7,8}$ Gene/protein-modified NF materials have the unique ability to interface with neural tissue at the nano-scale and are capable of influencing neuronal survival and neurovascular remodeling. In spinal cord injury, an extracellular matrix constructed of NF scaffolds, serving as a permissive bridge, affects glial scar formation and enhances axonal regeneration. ${ }^{9}$ Wang et al reported that glial cell-derived neurotrophic factor immobilized NF scaffolds promoted the survival, differentiation and maturation of implanted neural stem cells after transplantation into the brain parenchyma. ${ }^{9,10}$ In addition, an injectable functionalized nano-peptide underwent self-assembly to construct an interconnected network with intertwining NFs, and was used to promote sprouting angiogenesis and developmental neurogenesis in a zebrafish brain injury model. ${ }^{11}$

Vascular endothelial growth factor (VEGF) has recently been shown to be a potential therapeutic agent in ischemic central nervous system (CNS) disorders, including vascular dementia, focal cerebral ischemia, and cerebral ischemia- reperfusion injury. ${ }^{12}$ Hypoxic ischemia stimulates the secretion of VEGF and other angiogenic factors, leading to neovascularization and protection against ischemic injury. ${ }^{13}$ In a rat model of transient middle cerebral artery occlusion, VEGF-A promoted angiogenesis and neurogenesis through activating ERK1/2 pathways in the hippocampus and frontal cortex. ${ }^{14}$ However, the biological effects of endogenously up-regulated VEGF-A are limited due to its level and route of expression. Recently, many studies have reported that NF material embedded with VEGF-A can compensate for short action times. ${ }^{15}$ Biomaterial-mediated VEGF-A supplementation could help overcome the clinical dilemma of poor surgical revascularization results and provide protection from ischemic stroke. ${ }^{16,17}$ Furthermore, VEGF-A in a nanofibrous scaffold promotes regeneration of vascularized nerve tissue and functional recovery after spinal cord injury and cerebral ischemia/reperfusion injury. ${ }^{18,19}$ VEGF-A also links neurovascular coupling activity with angiogenesis and neurogenesis. ${ }^{20}$ Therefore, achieving an understanding of the molecular mechanisms by which VEGF-A coordinates neuronal homeostasis has become more urgent.

The endocannabinoid system (ECS) involves cannabinoid 1 and 2 receptors (CB1R and CB2R), two ligands (anandamide and 2-arachidonoylglycerol), and endogenous enzymes including fatty acid amide hydrolase (FAAH). FAAH inhibitors can increase the levels of endogenous analogs of cannabinoids or endocannabinoids and have been shown to protect against neuropathic pain and cannabis withdrawal symptoms, while some evidence suggests that FAAH inhibitors can lead to neuronal toxic syndromes in humans, such as headache, memory impairment, and altered consciousness. $^{21-23}$ 3'-Carbamoylbiphenyl-3-ylcyclohexylcarbamate (URB597), a potent and selective inhibitor of FAAH, strongly mediates ECS bioactivities. Growing preclinical work has shown URB597 could be a candidate for alleviating ischemic brain damage, as it could offer increased selective protection with less risk of the undesirable side effects that have been observed with CB agonists capable of activating all accessible receptors indiscriminately. ${ }^{23}$ URB597 decreases hippocampal microglial activation and the infarct volume in focal cerebral ischemia in mice. ${ }^{24}$ We previously reported the neuroprotective effects of URB597 against ischemic hippocampal neuronal loss and excessive autophagy damage. ${ }^{25,26}$ However, the molecular mechanisms by which URB597 regulates neuronal cells during OGD have not yet been systematically evaluated. 
To clarify these issues, in the present study, we investigated the potential effects of URB597 and VEGF-A embedded NF on primary hippocampal neurons, as well as the underlying mechanisms associated with its therapeutic potential under the condition of OGD.

\section{Methods and Materials}

\section{Materials and Reagents}

Poly(e-caprolactone) (PCL; $\mathrm{Mw}=80 \mathrm{kDa}, \quad$ No. 440752), dichloromethane and dimethyl sulfoxide (DMSO), and polyallylamine hydrochloride (PAH) hydrochloride $(1 \mathrm{mg} / \mathrm{mL}, \mathrm{pH}$ $=4.7$ ) and poly(sodium-styrenesulfonate) (PSS, $1 \mathrm{mg} / \mathrm{mL}, \mathrm{pH}$ = 4.7) were purchased from Sigma-Aldrich (St. Louis, MO, USA). Rabbit monoclonal antibodies against NSE (No. ab180943), Cleaved-poly-ADP ribose polymerase (PARP) (No. 94885), Cleaved-Caspase-3 (No. 9661), Bax (No. 14796), AKT (No. 9272), phospho-AKT (p-AKT) (No. 4060), CB1R, brain-derived neurotrophic factor (BDNF), tropomyosin receptor kinase B (TrkB), and beta Actin (Actin) (No. 4970) were from Cell Signaling Technology (Danvers, MA, USA). Anti-PI3K antibody (No. AF5112) and antip-PI3K antibody (No. AF7421) were from Affibiothech (Cincinnati, OH, USA). Anti-B-cell lymphoma-2 antibody (Bcl-2, No. ab196495) and JC-1 Mitochondrial Membrane Potential Assay Kit (No. ab113850) were from Abcam (Cambridge, MA, USA). Rat TNF- $\alpha$ (No. RTA00) and IL-1 $\beta$ (No. RLB00) and VEGF (No. DY564) ELISA Kits and recombinant rat VEGF-164 (VEGF-A, No. 564-RV) were from R\&D Systems, Inc (Minneapolis, MN, USA). Rat COX-2 (No. orb410912) and iNOS (No. orb411342) ELISA kits are from Biorbyt and Sigma-Aldrich (St. Louis, MO, USA). The Alexa 488/594-conjugated goat anti-rabbit/mouse antibody is from Invitrogen Carlsbad (CA, USA). A bicinchoninic acid BCA Kit was from Beyotime Biotechnology (Shanghai, China). In Situ Cell Death Detection Kit was from Promega Madison (WI, USA). URB597 (No. S2631) was purchased from Selleck (Houston, TX, USA). All culture medium was purchased from Gibco Life Technologies Co (Carlsbad, California).

\section{Fabrication of VEGF-A Loaded PCL NF Membrane}

A schematic image of the electrospinning technique is provided for PCL fiber membranes in Figure 1A as we previously reported. ${ }^{27}$ In brief, we prepared a polymer solution by dissolving PCL in a solvent mixture containing dichloromethane and DMSO $(3: 1, \mathrm{w} / \mathrm{w})$ at a concentration of $6 \%$ $(\mathrm{w} / \mathrm{w})$. Then, a $10-\mathrm{mL}$ glass syringe was used to collect the PCL electrospinning solution, which was loaded to a pump (LSP01-1A, Longer Pump Inc., Baoding, China). For NF generation, the flow rate of the solutions was controlled by the pump at $0.5 \mathrm{~mL} / \mathrm{h}$, a high voltage $(15 \mathrm{kV}, \mathrm{ZS}-60 \mathrm{kV} / 2$ $\mathrm{mA}$, Rixing Electric Inc. Shanghai, China) was applied to the needle tip, and the working distance between the needle tip and NF collector was $20 \mathrm{~cm}$. An aluminum foil collector (diameter $5 \mathrm{~cm}$ ) was used to collect the PCL NFs with rotation at $100 \mathrm{rpm}$. The environmental conditions of the spinning device were set at $35{ }^{\circ} \mathrm{C}$ and $30 \%$ humidity.

The VEGF-A loaded PCL NF membrane scaffold was fabricated in a layer-by-layer (LBL) self-assembly manner. ${ }^{28}$ Briefly, solutions of PAH $(1 \mathrm{mg} / \mathrm{mL})$ at $\mathrm{pH} 4.7$ and PSS $(1 \mathrm{mg} / \mathrm{mL})$ at $\mathrm{pH} 4.7$ were prepared. The LBL assembly process was divided into two steps. Firstly, the PCL membrane was immersed in the PAH solution for 1

A

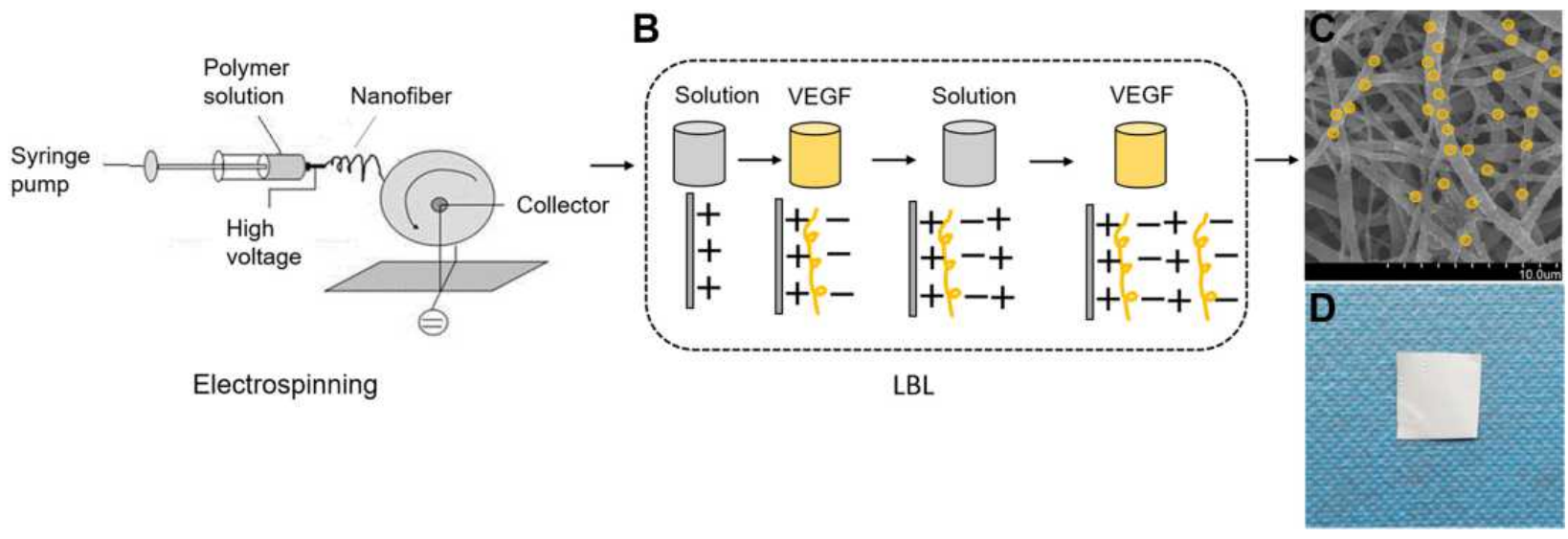

Figure I Schematic diagram of assembling VEGF-A-loaded PCL NF membrane. (A) Electrospinning. (B) Making the VEGF-A encapsulated PCL NF by the LBL method. (C) Representative SEM picture of VEGF-A NF (scale bar $10 \mu \mathrm{m})$. (D) VEGF-A NF product. 
$\mathrm{h}$ to form a positively charged precursor layer. Secondly, the PCL membrane was placed in a solution of negatively charged VEGF-A $(1 \mu \mathrm{g} / \mathrm{mL})$ for $5 \mathrm{~min}$. As shown in Figure $1 \mathrm{~B}$, the $\mathrm{LBL}$ process was repeated for 10 cycles to coat the PCL NFs with the VEGF-A loaded PAH/PSS multilayer film. For the control membrane, the PCL membrane was soaked in the solution mixture without VEGF following the formation of the PAH-derived positively charged precursor layer. $^{28}$ This dipping process was repeated for 5, 10, 15, and 20 cycles to make VEGF-A PCL in 5, 10, 15, and 20 multilayers.

\section{Characterizations of the NF Membranes}

The morphology of the NFs was observed using a scanning electron microscope (SEM) (S-4800, Hitachi Ltd., Tokyo, Japan). Each sample (0.5 g) was coated with gold (JFC-1600 Auto Fine Coater, Tokyo, Japan), placed in the vacuum chamber of the SEM, and scanned at an accelerating voltage of $20 \mathrm{kV}$. The average fiber diameter of each sample was measured using ImageJ software (NIH, Bethesda, MD, USA). An average of 100 fibers was counted for each sample. Chemical analysis of the composition of the NF membrane was conducted using an attenuated total reflection-Fourier transform infrared (ATR-FTIR) spectrometer (Tensor 27, Bruker, Woodlands, TX, USA). A wavelength range between 4000 and $400 \mathrm{~cm}^{-1}$ was recorded for the IR spectra at a resolution of $0.5 \mathrm{~cm}^{-1}$ with 40 scans per specimen.

The water contact angle (WCA) analysis was used to evaluate the hydrophilicity of the membranes. Each sample was cut into a $5 \times 5-\mathrm{cm}$ piece. Then, a single droplet of $1 \mu \mathrm{L}$ of deionized water was dropped on the surface of the sample, and the water-sample interaction was observed for $60 \mathrm{~s}$ through the Model 200 video-based optical system (Future Scientific Ltd Co., Taiwan, China). ${ }^{29}$ The average WCA of each group was calculated using the VCA Optima software (VCA3000S, AST, Pittsburgh, PA, USA).

The loaded VEGF-A bioactivity of NFs with different layers was detected using a VEGF-A ELISA Kit (R\&D). The membranes of each group were cut into $1 \times 1-\mathrm{cm}$ pieces, accurately weighed, and soaked in $10 \mathrm{~mL}$ of phosphate-buffered saline (PBS) at $37^{\circ} \mathrm{C}$. The samples were collected and placed into the same amount of fresh PBS at the same times each day. The percentage of VEGF-A released was assessed according to the instructions of the ELISA Kit (St. Louis, MO, USA). Testing was performed using a PerkinElmer Victor 3 multilabel reader with a 405- $\mathrm{nm}$ filter and 650-nm correction filter, and was repeated three times daily for 35 days.

\section{Culture of Primary Hippocampal Neurons}

Hippocampal neurons were isolated from embryonic day 18 (E18) Sprague-Dawley rats and cultured as described previously. ${ }^{30}$ Briefly, embryos were decapitated after cleaning with $75 \%$ alcohol. The hippocampus without meninges was carefully extracted from the brain under a microscope (Olympus, Tokyo, Japan) and transferred to an ice-cold buffer composed of $127 \mathrm{mM} \mathrm{NaCl}, 1.7 \mathrm{mM}$ $\mathrm{NaH}_{2} \mathrm{PO}_{4}, 5 \mathrm{mM} \mathrm{KCl}, 2.05 \mathrm{mM} \quad \mathrm{KH}_{2} \mathrm{PO}_{4}, 10 \mathrm{nM}$ D-glucose, and $100 \mathrm{U} \mathrm{mL}^{-1}$ penicillin/streptomycin $(\mathrm{pH}$ 7.4). Shredded hippocampus tissues were dissociated using the Neuron Isolation Enzyme Kit (with papain) (Thermo Fisher, MA, USA), and then incubated at $37{ }^{\circ} \mathrm{C}$ for 10 min. The tissue solution was obtained following a wash with Hank's Balanced Salt Solution (Gibco, MA, USA), collected on a 100-mesh grid, and centrifuged at $1000 \mathrm{rpm}$ for $5 \mathrm{~min}$. After centrifugation, the supernatant was discarded. Primary rat neurons were resuspended in a minimum essential medium composed of $1 \%$ GLUTMAX and 2\% B-27 Supplement (both from Gibco, MA, USA). A sufficient volume of the cell suspension was transferred to a petri dish containing poly-L-lysine-coated coverslips for culture at $37{ }^{\circ} \mathrm{C}$ and $5 \% \mathrm{CO}_{2}$ in a sterile incubator.

\section{Identification of Neurons}

After culturing, the purity of the hippocampal neuron was detected with neuron-specific enolase (NSE) overnight by immunofluorescence staining. Briefly, cells were fixed in $4 \%$ paraformaldehyde for $20 \mathrm{~min}$. Cells were washed twice with PBS, permeabilized with $0.1 \%$ Triton X-100 (Sigma-Aldrich) in PBS for $5 \mathrm{~min}$, and then blocked with 5\% goat serum (Sigma-Aldrich) in PBS for $1 \mathrm{~h}$. Subsequently, cells were incubated overnight at $4{ }^{\circ} \mathrm{C}$ with anti-NSE (1:300) antibody. Then they were washed with PBS and incubated with Alexa Fluor 488-conjugated goat anti-rabbit IgG (1:500, Jackson, West Grove, PA, USA) at $37{ }^{\circ} \mathrm{C}$ for $1 \mathrm{~h}$. Coverslips were mounted to glass slides using ProLong Gold antifade reagent containing 4' 6-diamidino-2-phenylindole (DAPI) and imaged using a fluorescence microscope (Zeiss, Jena, Thuringia, Germany). 


\section{Oxygen-Glucose Deprivation (OGD) and Treatment Groups}

Primary hippocampal neurons were cultured for OGD as previously described. ${ }^{31}$ Their density was $7 \times 10^{5}$ cells/well in 6-well plates. Cells were transferred from the Neurobasal-A medium to DMEM without glucose (Gibco, USA) in 6-well plates. In this medium, cells were incubated at $37{ }^{\circ} \mathrm{C}$ for $4 \mathrm{~h}$ in a sealed chamber with an anaerobic gas mixture $\left(5 \% \mathrm{CO}_{2}\right.$ and $\left.95 \% \mathrm{~N}_{2}\right)$. Neurons cultured without OGD were used as a negative control (the control group). Neurons cultured under OGD with a blank NF membrane without VEGF-A-loaded were the a VEGF-A control (the VEGFC group). After $4 \mathrm{~h}$ of OGD, cells were incubated in a maintenance medium for $72 \mathrm{~h}$ under normal conditions prior to subsequent experiments. Cells in the maintenance medium with an equal amount of solution (excluding URB597) were set as a URB597 control (URBC). Cell treatment groups were as follows: (1) the control group (the Con group), (2) the OGD group (the OGD group), (3) the URB597 control group (the URBC group), (4) the URB597 (5.68 $\mu$ mol) treatment group (the URB group), (5) the VEGF-A control group (the VEGFC group), (6) the VEGF-A treatment group (the VEGF group), (7) the URB597 and VEGF-A co-treatment group (the URB+VEGF group).

\section{Cell Morphology Scanning}

The morphology of the neurons cultured on the NFs was determined from SEM scans. Briefly, the neurons in different groups were cultured on the NFs for $72 \mathrm{~h}$. The cells were immersed in PBS containing 1\% osmic acid for 4 $\mathrm{h}$ following immersion in PBS containing 2.5\% glutaraldehyde. Then, the cells were washed thrice with PBS. The samples were placed sequentially in ethanol solutions at concentrations of $20 \%, 40 \%, 60 \%, 80 \%$, and $100 \%$ for dehydration. After drying at $25{ }^{\circ} \mathrm{C}$ for $1 \mathrm{~h}$, the samples were gold-sputtered under vacuum and visualized using an SEM (S-4800, Hitachi Ltd., Tokyo, Japan). The cell adhesion rate, the remaining amount of cell divided by inoculation amount of cell, was counted by an investigator blinded to the groups.

\section{Cell Viability Assay}

Equal number of cells (at a density of $1 \times 10^{5}$ neurons $/ \mathrm{mL}$ ) from six samples taken from each group were seeded in a 96-well plate. Neuron viability was assessed by 3-(4,5-dimethyl-thiazol-2-yl)-2, 5-diphenyl-tetrazolium bromide (MTT) (Sigma, USA) assay. We added $20 \mu \mathrm{L}$ MTT solution ( $5 \mathrm{mg} / \mathrm{mL}$ MTT in PBS, pH 7.4) to each well at $12 \mathrm{~h}, 24 \mathrm{~h}, 48 \mathrm{~h}, 72 \mathrm{~h}$, and $96 \mathrm{~h}$ following different interventions. Then, the plate was incubated for $4 \mathrm{~h}$ at $37^{\circ}$ $\mathrm{C}$ and $5 \% \mathrm{CO}_{2}$ in a sterile incubator. After removing the medium, DMSO was added to dissolve the resulting crystals. Finally, a plate reader was used to read the absorbance (OD) at $490 \mathrm{~nm}$.

\section{Reactive Oxygen Species (ROS) and Mitochondrial Membrane Potential (MMP,} $\Delta \psi \mathrm{m})$ Measurement

The hippocampal neurons were placed in 96-well plates at a density of $1 \times 10^{4} /$ well, and were cultured for $72 \mathrm{~h}$ and used for measuring ROS concentrations via an ROS Assay Kit (Beyotime, Shanghai, China). Cellular ROS levels were evaluated using the oxidant-sensitive probe 2,7-dichlorofluorescein diacetate (DCF-DA) according to the manufacturer's instructions. Firstly, cells were centrifuged at $1000 \mathrm{rpm}$ for $5 \mathrm{~min}$ following washing with PBS. Then, cells were incubated with DCF-DA solution at $37^{\circ}$ $\mathrm{C}$ and $5 \% \mathrm{CO}_{2}$ for $30 \mathrm{~min}$. A fluorometric microplate reader (FilterMax F5, Molecular Devices, Sunnyvale, USA) with excitation and emission at 485 and $530 \mathrm{~nm}$, respectively, was used to measure the mean fluorescence intensity (MFI). MFI served as an indicator of the relative levels of mitochondrial oxidative stress.

Similarly, cells in different groups were cultured for 72 $\mathrm{h}$ and used to test $\Delta \psi \mathrm{m}$. According to the instruction of the JC-1 Kit (Beyotime, Shanghai, China), cells were washed with PBS, and incubated with JC-1 staining solution at 37 ${ }^{\circ} \mathrm{C}$ and $5 \% \mathrm{CO}_{2}$ for $20 \mathrm{~min}$. Afterward, areas of red and green fluorescence were captured using a fluorescence microscope (Zeiss, Jena, Thuringia, Germany). The ratio of the red/green fluorescence was calculated by an investigator blinded to the groups.

\section{TdT-Mediated dUTP Nick End Labeling (TUNEL) Assay}

Neurons were processed with the In Situ Cell Death Detection Kit (Promega, Madison, WI, USA) to assess the number of apoptotic cells according to the manufacturer's instruction. Cells were fixed by $4 \%$ paraformaldehyde for $20 \mathrm{~min}$, and permeabilized with $0.1 \%$ Triton X-100 (Sigma-Aldrich) in PBS for 5 min. $50 \mu \mathrm{L}$ TUNEL reaction reagent were added to each sample. Cells were stained by DAPI for $5 \mathrm{~min}$ following incubation at $37^{\circ} \mathrm{C}$ 
for $1 \mathrm{~h}$ in the dark. TUNEL-positive cells were counted at $200 \times$ magnification in four non-overlapping microscope fields (Zeiss, Jena, Thuringia, Germany). The rate of apoptosis was calculated as the ratio of TUNEL-positive cells (green) to total cells (DIPA, blue).

\section{Western Blotting}

Hippocampal neuron protein concentrations were determined using a BCA Kit (Beyotime, Shanghai, China). Equal amounts of neuronal cell protein $(30 \mu \mathrm{g})$ were separated by 6-12\% sodium dodecyl sulfatepolyacrylamide gel electrophoresis and transferred to a polyvinylidene difluoride (PVDF) membrane. The PVDF membrane was blocked using 5\% non-fat milk at $37{ }^{\circ} \mathrm{C}$ for $1.5 \mathrm{~h}$. Subsequently, the PVDF membrane was incubated with primary antibodies against CB1R (1:1000), PI3K (1:1000), p-PI3K (1:1000), Akt (1:1000), p-Akt (1:1000), BDNF (1:500), TrkB (1:1000), Caspase-3 (1:500), Bcl-2, (1:500), PARP (1:1000), Bax (1:1000), and Actin $(1: 5000)$ at $4^{\circ} \mathrm{C}$ for $24 \mathrm{~h}$. After washing three times, the PVDF membranes were incubated with secondary antibodies conjugated with horseradish peroxidase at room temperature for $1 \mathrm{~h}$. The Western blotting bands were visualized using the enhanced chemiluminescence method, and then quantified by ImageJ software (NIH, Bethesda, MD, USA) by an investigator blinded to the groups.

\section{Enzyme-Linked Immunosorbent Assay (ELISA)}

The cell culture supernatants were collected after centrifugation and stored at $-80^{\circ} \mathrm{C}$. The levels of proinflammatory cytokines TNF- $\alpha$, IL-1 $\beta$, COX-2, and iNOS were measured using the relevant ELISA Kits (Sigma-Aldrich, St. Louis, MO, USA). The protocols were performed according to instructions provided by the manufacturer. All measurements were repeated three times and were performed by two experimenters blinded to the details of the group.

\section{Reverse Transcription-Polymerase Chain Reaction (RT-PCR)}

Total RNA was extracted with TRIzol reagent (Invitrogen, Carlsbad, CA, USA) following the manufacturer's instructions. RNA (1000 ng) was placed into a $20-\mu \mathrm{L}$ reaction system for reverse transcription with the Prime Script RT Reagent Kit (Toyobo, Osaka, Japan). cDNA synthesis and amplification were performed using the SYBR Green PCR Kit (Toyobo, Osaka, Japan) on a real-time PCR System (Applied Biosystems 7500). The PCR primers were designed by and purchased from Sangon Biotech (Shanghai, China) (Table 1). The PCR reaction cycles were as follows: denaturation for $30 \mathrm{~s}$ at $95^{\circ} \mathrm{C}, 40$ cycles of amplification ( $5 \mathrm{~s}$ at 95 ${ }^{\circ} \mathrm{C}, 30 \mathrm{~s}$ at $60{ }^{\circ} \mathrm{C}$, and $30 \mathrm{~s}$ at $72{ }^{\circ} \mathrm{C}$ ), annealing for $5 \mathrm{~min}$ at $72{ }^{\circ} \mathrm{C}$. The relative expression level of each gene was calculated using the $2^{-\Delta \Delta \mathrm{Ct}}$ method as previously reported. ${ }^{28,32}$

\section{Statistical Analysis}

The data are expressed as the mean \pm standard deviation (SD). The significances among multiple groups were determined by one-way analysis of variance (ANOVA) followed by a Bonferroni post hoc test using SPSS Statistics 22.0 statistical software (SPSS Inc., Chicago, IL, USA). Comparison between two groups were

Table I Primer Sequences for RT-PCR

\begin{tabular}{|l|l|l|}
\hline Gene & Primer & Length \\
\hline PARP & $\begin{array}{l}\text { Upstream: 5'-TCGACGTGGAGAGCATGAAG-3' } \\
\text { Downstream: 5'-ATAGAGTAGGCGGCCTGGAT-3' }\end{array}$ & 106bp \\
\hline Caspase-3 & $\begin{array}{l}\text { Upstream: 5'-GAGCTGGACTGCGGTATTGA-3' } \\
\text { Downstream: 5'-TAGTAACCGGGTGCGGTAGA-3' }\end{array}$ & II 3bp \\
\hline Bcl-2 & $\begin{array}{l}\text { Upstream: 5'-ACTCTTCAGGGATGGGGTGA-3' } \\
\text { Downstream: 5'-TGACATCTCCCTGTTGACGC-3' }\end{array}$ & 94bp \\
\hline Bax & $\begin{array}{l}\text { Upstream: 5'- CATGTTT GCAGACGGCAACT-3' } \\
\text { Downstream: 5'-TGATCAGCTCGGGCACTTTA-3' }\end{array}$ & 107bp \\
\hline Actin & $\begin{array}{l}\text { Upstream: 5'-GGAGAAGATTTGGCACCACAC-3' } \\
\text { Downstream: 5'-ACACAGCCTGGATGGCTACG-3' }\end{array}$ & I73bp \\
\hline
\end{tabular}


performed by Student's $t$-test. Differences with $p$ values less than 0.05 were considered statistically significant.

\section{Results}

\section{Synthesis and Characterization of NFs}

As shown in Figure 1A, the random PCL NF membrane was fabricated using the electrospinning technique. The LBL selfassembly method was used to successfully coat the PCL NFs with VEGF-A (Figure 1B-D). We made four different membranes with 5, 10, 15, and 20 layers of VEGF-A NF. Then, we estimated the physical, chemical, and bioactive properties of VEGF-A loaded layered PCL membranes. We screened for the best VEGF-A NF membranes by using SEM, ATR-
FTIR spectroscopy, the WCA test, VEGF-A release profile and, MTT cell survival measurements.

The microscopic morphologies of different LBL structure scaffolds were observed by SEM. Figure 2A shows a smooth surface with no observable particulate matter in the PCL fiber. As VEGF-A was assembled on the NFs, the surface topography of VEGF-A NFs in different layers began to change. As the number of layers increased, particulate matter on the surface could be observed. The 5- and 10-layer of VEGF-A NFs had randomly interconnected micropores and were highly uniform. Compared to those, there was too much obvious particulate matter in the 15- and 20-layer of VEGF-A NFs, which gradually blocked the micropores. In addition, according to the distribution of

A
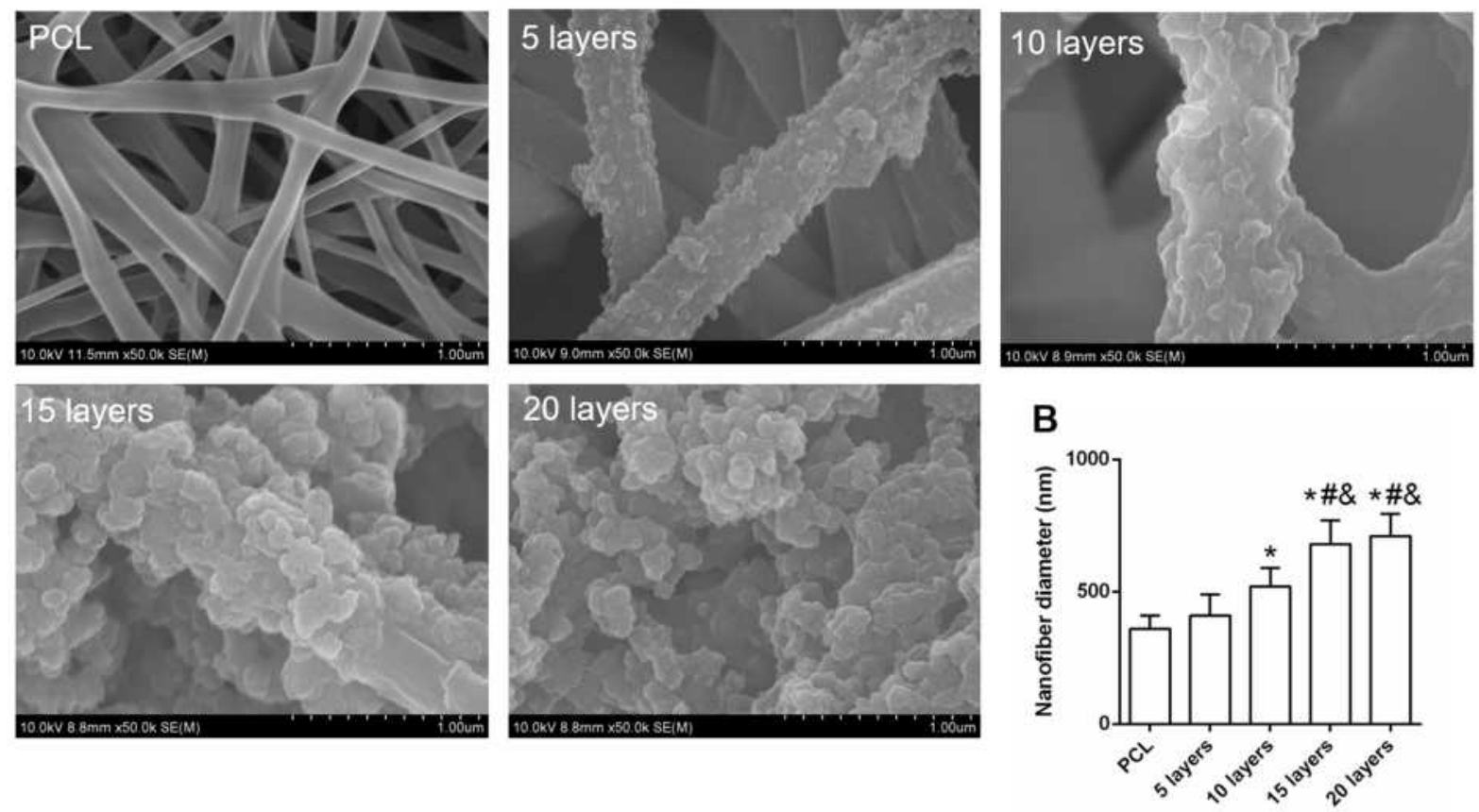

C

D

E
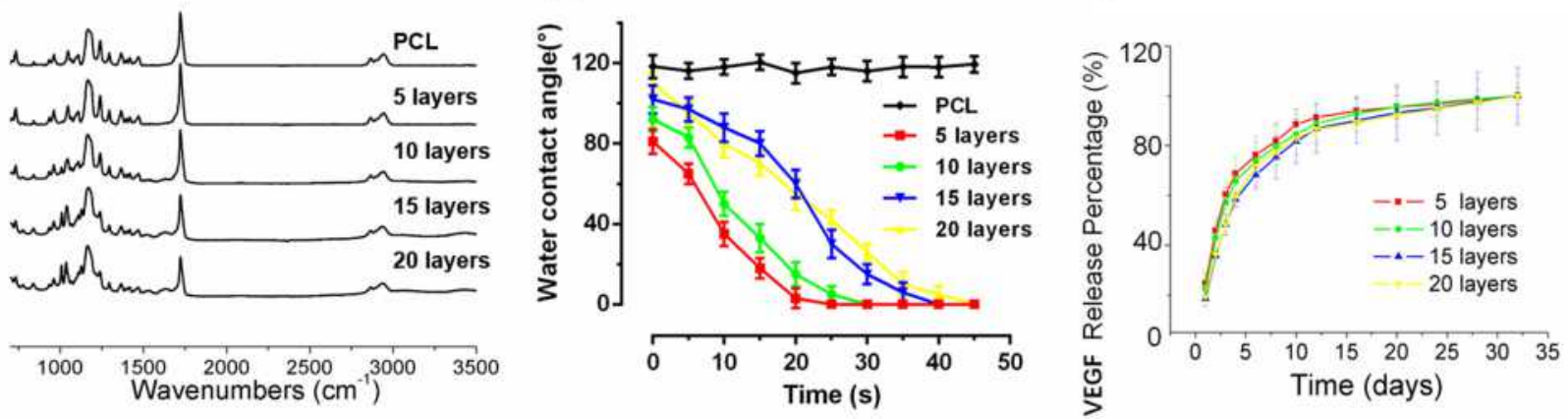

Figure 2 Characterization of PCL electrospun NFs with different self-assembly layer. (A) SEM images. (B) The average diameter of NFs in different layers. (C) FTIR spectra of differently coated PCL surfaces of 5-, 10-, 15-, and 20-layer (D) Water contact angle curves decaying with time (E) The controlled release curves of VEGF-A from different $\mathrm{PCL}$ membranes in vitro. ${ }^{*} \mathrm{P}<0.05$ vs $\mathrm{PCL}$; ${ }^{\#} \mathrm{P}<0.05$ vs 5 layers; ${ }^{\&} \mathrm{P}<0.05$ vs 10 layers. 
fiber diameters, the average diameter in the 5-layer VEGF-A NFs group was similar to that of the PCL group. The diameters of NFs in other groups increased significantly ( $\mathrm{p}<0.05,10$ layers vs PCL; $\mathrm{p}<0.05,15$ layers vs PCL; $\mathrm{p}<$ 0.05, 20 layers vs PCL, respectively) (Figure 2B), and there was no significant difference between NFs with 15 and 20 layers. The SEM results revealed that all samples maintained a nanofibrous 3D structure.

As shown in Figure 2C, we further detected the ATRFTIR spectra of the PAH/PSS multilayer coating-modified PCL scaffold. The peak between 1140 and $1250 \mathrm{~cm}^{-1}$ corresponds to the absorption of the stretching vibration of the C-C bond. The vibration spectrum between 1390 and $1500 \mathrm{~cm}^{-1}$ are closely related to the $\mathrm{C}-\mathrm{H}$ bond. Because of the asymmetric stretching vibration of the $\mathrm{SO}_{3}$ group in PSS, there are distinct absorption bands at $1080 \mathrm{~cm}^{-1}$ and $1130 \mathrm{~cm}^{-1}$ in the ATR-FTIR spectrum. Furthermore, this spectrum also shows an absorption peak at $1610 \mathrm{~cm}^{-1}$, which is due to an amide group in PSS. These results proved that the PAH/PSS multilayer was successfully assembled on the PCL scaffold.

To analyze the hydrophilicity of nanofibrous membranes with different layers, the water contact angle was detected. As shown in Figure 2D, the initial water contact angles of 5-, 10-. 15- and 20-layer NF membranes ranged from $80.5^{\circ}$ to $106.5^{\circ}$. As the number of layers increased, the initial water contact angle also increased. The water droplets were completely absorbed within $50 \mathrm{~s}$ in the multilayer groups, while that of the PCL group could maintain a high angle $\left(116.5 \pm 2^{\circ}\right)$ for a long time. There was no significant difference in the time it took for the water droplet to be absorbed between the 5-layer group and the 10-layer group. The times of the absorption in the 15- and 20-layer groups were similar, which were significantly different from and higher than the absorption time for the 5-layer and 10-layer groups. These data indicated that the hydrophilicity of the nanofibrous membranes was significantly increased after coating with the PAH/PSS complex. However, when the number of LBL multilayers exceeded 15, the hydrophilicity of the membranes was reduced, similar to the results of a previous study. ${ }^{29}$

The release profiles of VEGF-A in NFs of different layers were investigated using an ELISA Kit. As presented in Figure 2E, there was an initial burst release from all the different layers during the first 3 days. The percentages of VEGF-A released from the 5-, 10-, 15-, and 20-layer membranes were $24.7 \%, 22.1 \%, 18.3 \%$, and $20.5 \%$, respectively, in the beginning. On day 3 , the percentage of VEGF-A released from the 5-, 10-, 15-, and 20-layer membranes were $60.4 \%, 53.7 \%, 48.3 \%$, and $48.3 \%$, respectively. After 10 days, $89.5 \%$ of VEGF-A was released from the 5-layer membrane, and $83.2 \%$ of VEGF-A was released from the 10-layer membrane. The released rates of VEGF-A from the 15-layer membrane and the 20-layer membranes were similar, about $81 \%$. However, in the 5-layer membranes, the release rate of VEGF-A reached a plateau quickly (Figure 2E), whereas the release rate in the 10-layer membranes was somewhere between those of the 5-layers and 15-layer membranes. Considering the differences in diameters and morphologies (Figure 2A and B), the 10-layer VEGF-A NF membrane may be considered appropriate for subsequent experiments. Moreover, a subsequent stable release of VEGF-A for up to 35 days was detected, indicating that the 10-layer VEGF-A NF membrane is capable of maintaining a longterm release of VEGF-A.

\section{VEGF-A NF Membrane and URB597 Promote Cell Adhesion, Proliferation, and Viability During OGD}

NSE immunofluorescence staining suggested that primary cultured hippocampal neurons were successfully obtained (Figure 3). To estimate the biocompatibility of the NF membrane, the impact of VEGF-A and URB597 on cell adhesion and growth during OGD were assessed. Cell morphologies and growth were detected by SEM and MTT, respectively. As shown in Figure 4A, the nanofibrous scaffolds in different groups similarly supported the attachment and growth of neurons while different cell morphologies were displayed. In the OGD, URBC, and VEGFC groups, the cells on the surface of the NF adhered sparsely with a spherical shape. However, the neurons formed dense structures that grew on and migrated into the pores of the PCL NFs after URB597 or VEGF-A NF treatment, and especially in the UBR+VEGF treatment group. The cells were strongly attached to the NF surfaces almost in a cluster. Statistical results showed that the cell adhesion rates in the OGD, URBC, and VEGFC groups were lower than that in the Con group (all $\mathrm{p}<0.05$, vs Con group) (Figure 4B). Compared with the OGD group, URB597 treatment and VEGF-A NF treatment significantly increased the cell adhesion (all $\mathrm{p}<0.05$ ). In addition, the cell adhesion rate in either the URB597 group or the VEGF group was lower than that in the URB+ VEGF group $(\mathrm{p}<0.05)$. 

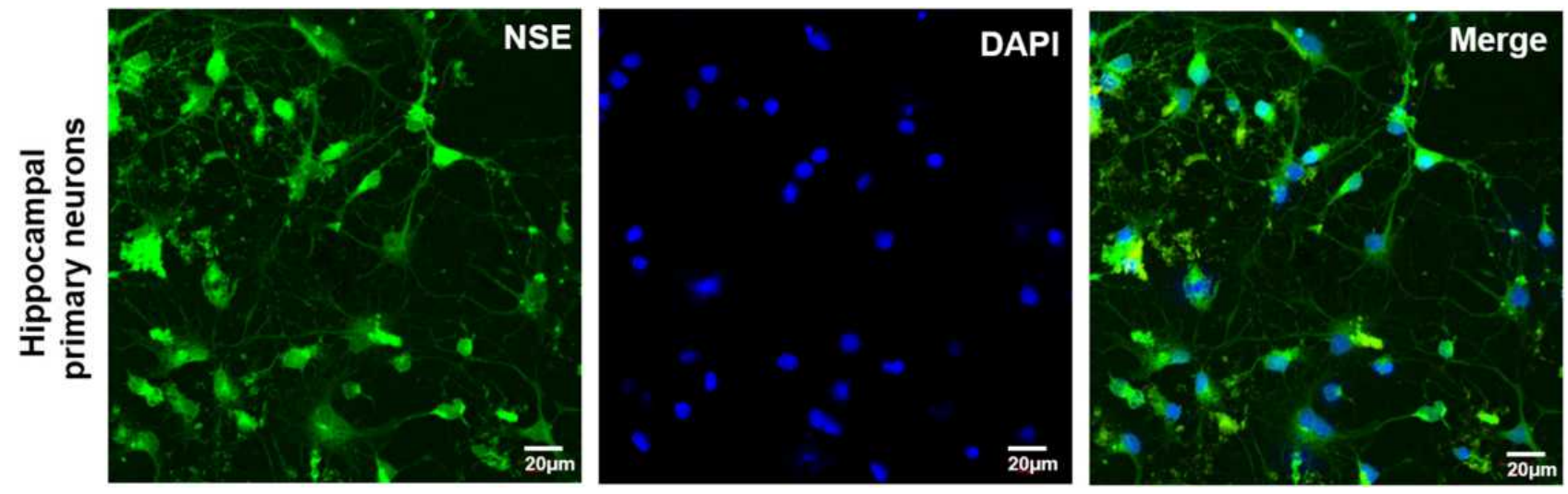

Figure 3 Representative NSE (green) immunofluorescence for neurons. Magnificent: $200 \times$, scale bar $20 \mu \mathrm{m}$ ).

Neuronal survival and growth were estimated using MTT. As shown in Figure 4C, the OD values were detected at $12 \mathrm{~h}, 24 \mathrm{~h}, 48 \mathrm{~h}, 72 \mathrm{~h}$, and $96 \mathrm{~h}$ respectively. OGD caused a decline in cell survival and growth with OD values decreasing during culture. Neuron proliferations in the URB group, VEGF group, and URB+VEGF groups were found to be better than that in the OGD group, especially at the time of $72 \mathrm{~h}(\mathrm{p}<0.05$, respectively). Taken together, these results suggest that UBR597 and VEGF-A promoted the survival and growth of neurons in vitro. Moreover, the ability of the VEGF-A loaded NF membranes to support neuron adhesion was proved.

\section{VEGF-A NF Membrane and URB597 Inhibit OGD-Induced Apoptosis}

As shown in Figure 5A, the number of TUNEL-positive cells (green fluorescence signal) in the OGD group was markedly larger than that in the control group $(\mathrm{p}<0.05)$. There was no difference between the URBC group and the VEGFC group. However, VEGF-A NF membrane treatment and URB597 treatment significantly decreased the positive cells (all $p<0.05$, vs OGD group). Green fluorescence was partially downregulated in the URB+VEGF group compared with the URB group and the VEGF group (all $\mathrm{p}<0.05$ ) (Figure 5B), indicating VEGF-A and URB597 could mitigate the TUNEL-positive cellar apoptosis levels.

We further investigated the changes in neuronal apoptosis-related genes, such as PARP, cleaved-Caspase-3, Bax, and Bcl-2, using Western blotting and RT-PCR (Figure 5C). Neurons that had experienced OGD showed high levels of PARP, cleaved-Caspase-3, Bax, and low levels of Bcl-2 in protein and mRNA (Figure 5D-J). Those enhanced expressions were reversed by URB597 treatment, VEGF-A NF membrane treatment, and the combination treatment (all $\mathrm{p}<0.05$, vs OGD group). Similarly, the change of Bcl-2 was also reversed (Figure 5C and J) (all $\mathrm{p}<0.05$, vs OGD group). Combined with the above TUNEL findings, these results suggest that the VEGF-A NF membrane and URB597 could suppress OGD-induced neuronal apoptosis.

\section{VEGF-A NF Membrane and URB597 Attenuate OGD-Induced MMP Injury}

The cyanine dye JC-1 was used to visualize the populations of mitochondria with a high MMP (red fluorescence) and low MMP (green fluorescence) (Figure 6A). Therefore, the ratio between the red and green fluorescence of cells loaded with JC-1 is often used for assessing mitochondrial damage. OGD caused a significant decrease in the MMP of neurons $(\mathrm{p}<$ 0.05, vs Con group). However, URB597 treatment, VEGF-A NF treatment, and URB597 combined with VEGF-A NF treatment notably alleviated the decrease in OGD-induced MMP (all p $<0.05$, vs OGD group) (Figure 6B). Furthermore, combination treatment was more effective than single treatment $(\mathrm{p}<0.05$, vs URB+VEGF group) (Figure 6B). No differences were observed among the OGD group, URBC group, and VEGFC group. These results indicated that the VEGF-A NF membrane and URB597 treatment improved OGD-induced mitochondrial injury, as they have synergistic protective effects on MMP.

\section{VEGF-A NF Membrane and URB597 Suppress OGD-Induced Neuronal Oxidative Stress and Inflammatory Response}

Mitochondria are the main sources of intracellular ROS. It remained unclear whether VEGF-A and URB597 were 
A

TEM
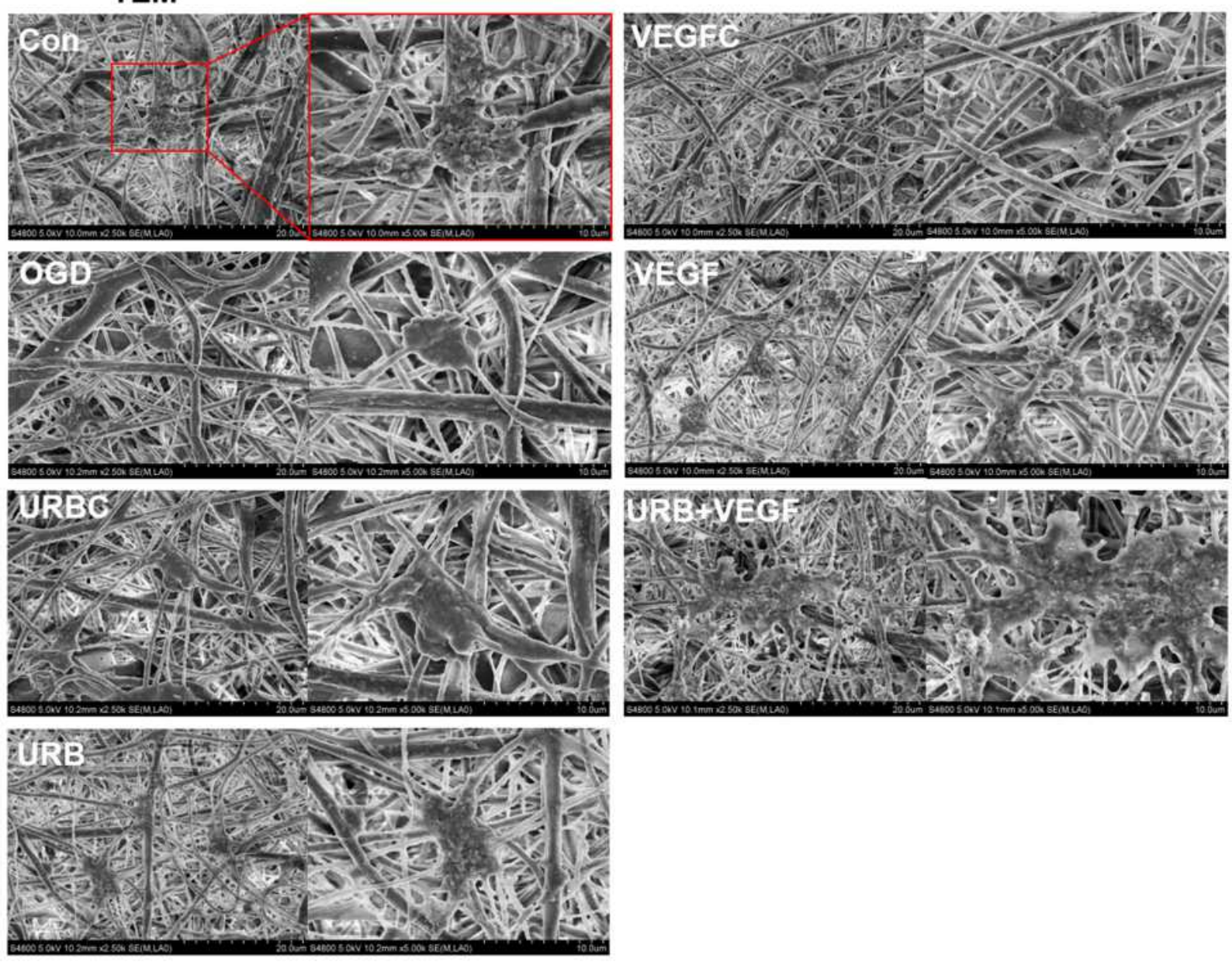

B

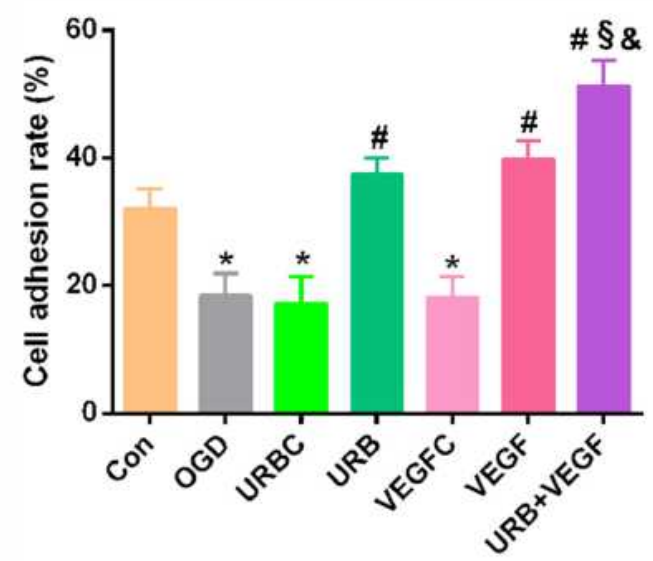

C

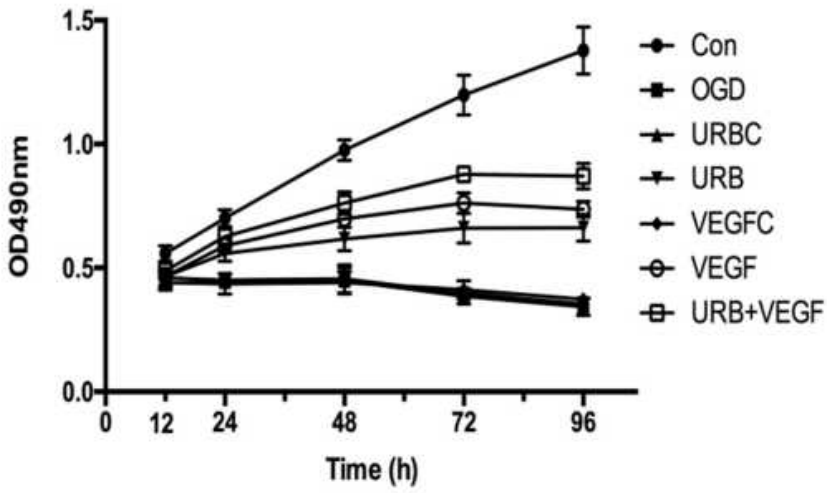

Figure 4 Effects of VEGF-A NF membrane and URB597 on cell morphology and viability. (A) Typical images of hippocampal neurons in PCL membrane by SEM (scale bar 20 and $10 \mu \mathrm{m}$ ). (B) Statistical results of the cell adhesion rate (CAR) in groups. (C) MTT assay showing neuron proliferation on OD values. ${ }^{* P}<0.05$ vs Con; ${ }^{\#} P<0.05$ vs $O G D$; ${ }^{\S} \mathrm{P}<0.05$ vs URB; ${ }^{\circledR} \mathrm{P}<0.05$ vs VEGF. 
A

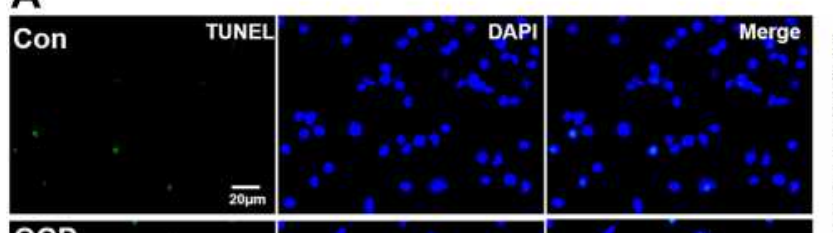

OGD

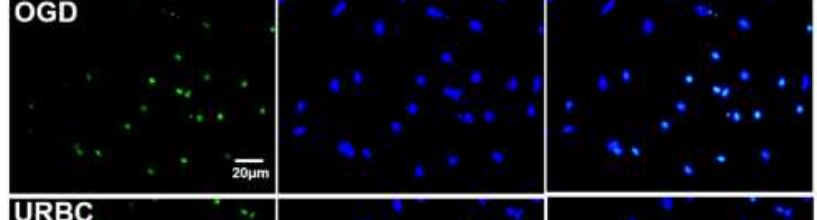

URBC,

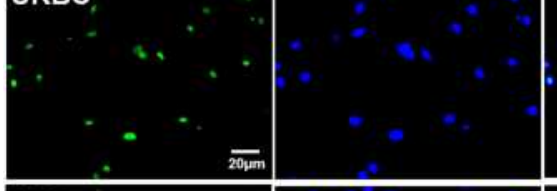

\section{URB}
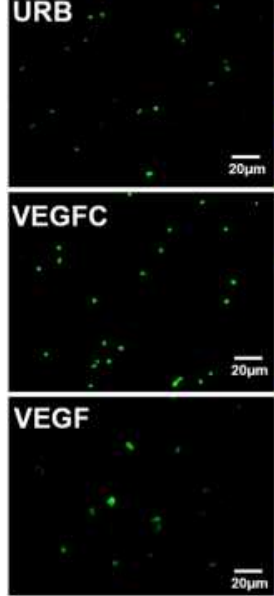

URB+VEGF
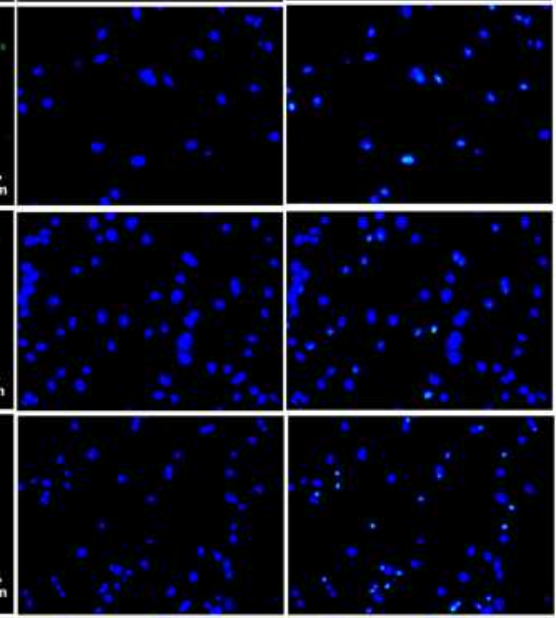

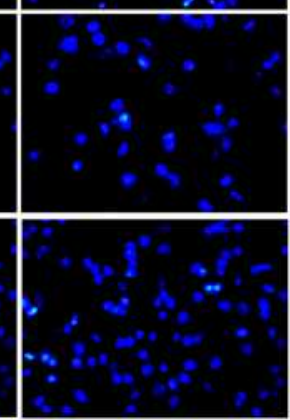

B
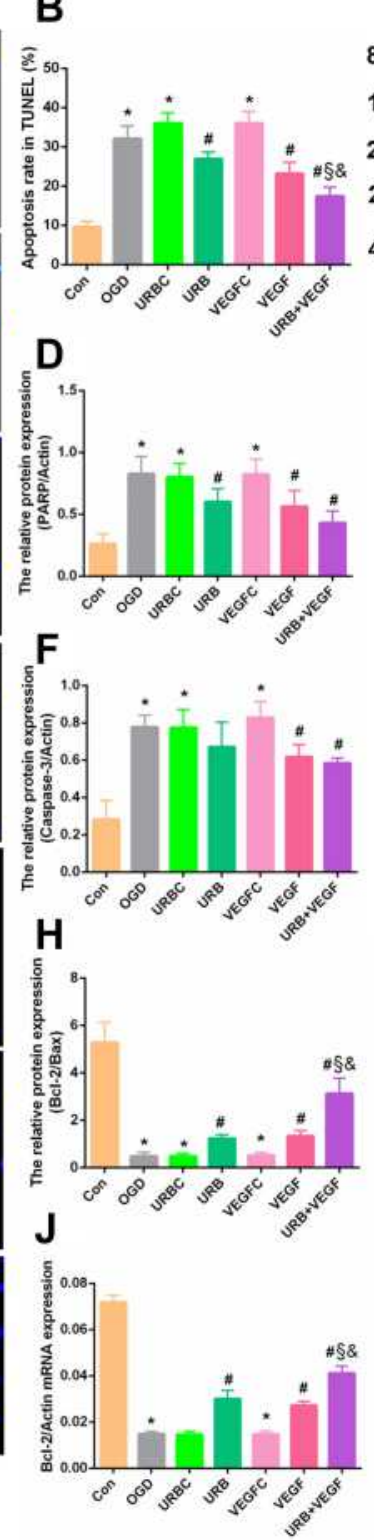

C

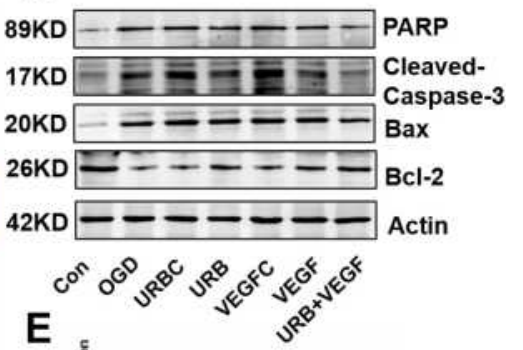

E

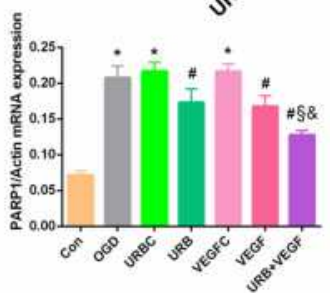

G
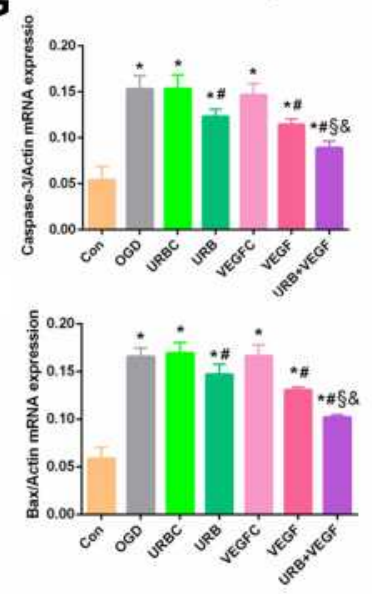

Figure 5 Effects of VEGF-A NF membrane and URB597 on apoptosis. (A) Representative TUNEL staining in neurons. (B) Quantitative analysis of the apoptosis rate in TUNEL. (C) Representative Western blot of apoptosis-related protein. (D-G) Quantitative analysis of the apoptosis proteins. (H-J) Quantitative analysis of mRNAs of apoptosis-related genes. ${ }^{* P}<0.05$ vs Con; ${ }^{\#} \mathrm{P}<0.05$ vs OGD; ${ }^{\S} \mathrm{P}<0.05$ vs URB; ${ }^{\circledR} \mathrm{P}<0.05$ vs VEGF.

involved in the oxidative stress response under the condition of OGD. The data obtained from the flow cytometry analysis showed that the intracellular ROS increased significantly following OGD $(\mathrm{p}<0.05$, vs Con group) (Figure 7A and B). After URBC treatment or VEGFC treatment, there was no obvious change in the level of ROS compared with the OGD group (all $\mathrm{p}>$ 0.05). In contrast, the level of ROS decreased significantly after URB treatment, VEGF-A NF membrane treatment, and combination therapy (all p $<0.05$, vs OGD group), indicating VEGF-A and URB597 mitigated the ROS levels. In addition, the ROS level in the combination therapy group was lower levels than those in the VEGF group and the URB group ( $p<0.05$, respectively), and had the optimal effectiveness in suppressing ROS (Figure 7B). Therefore, these data confirmed that VEGF-A and URB597 inhibited OGD-induced ROS production and the resultant oxidative stress response.

Several theories have been proposed to explain the cellular ROS production responsible for the activation of neuroinflammation. ${ }^{33}$ A robust inflammatory response induced by cerebral ischemia plays a critical role in 


\section{A}
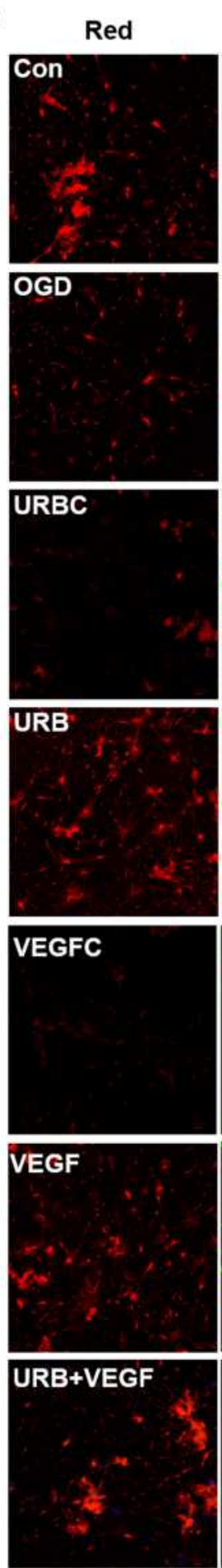

Green
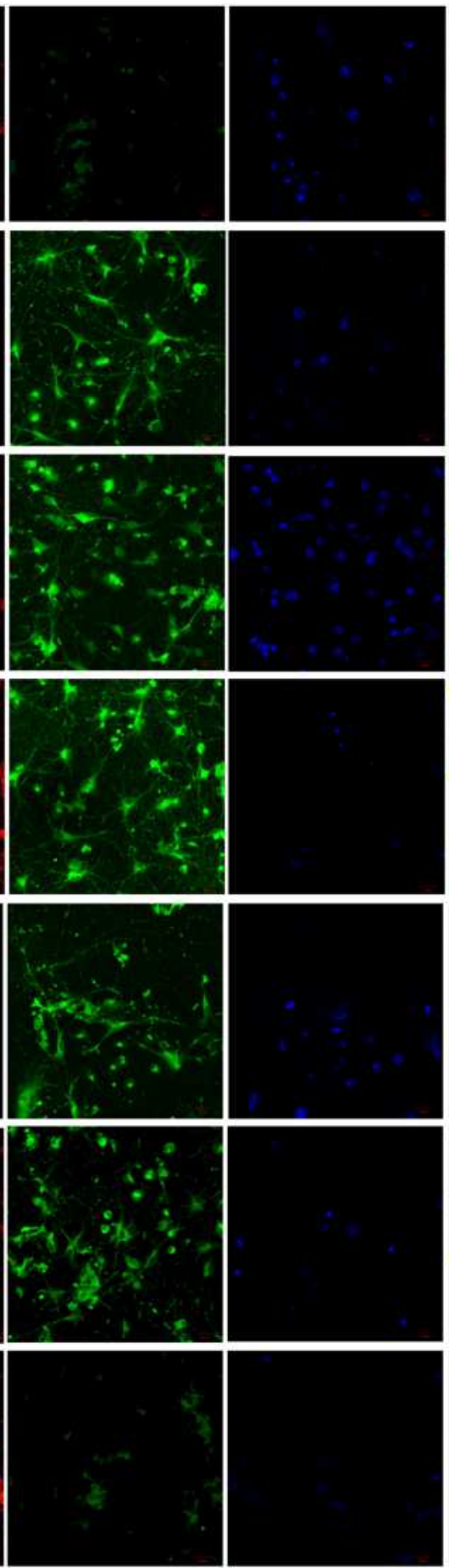
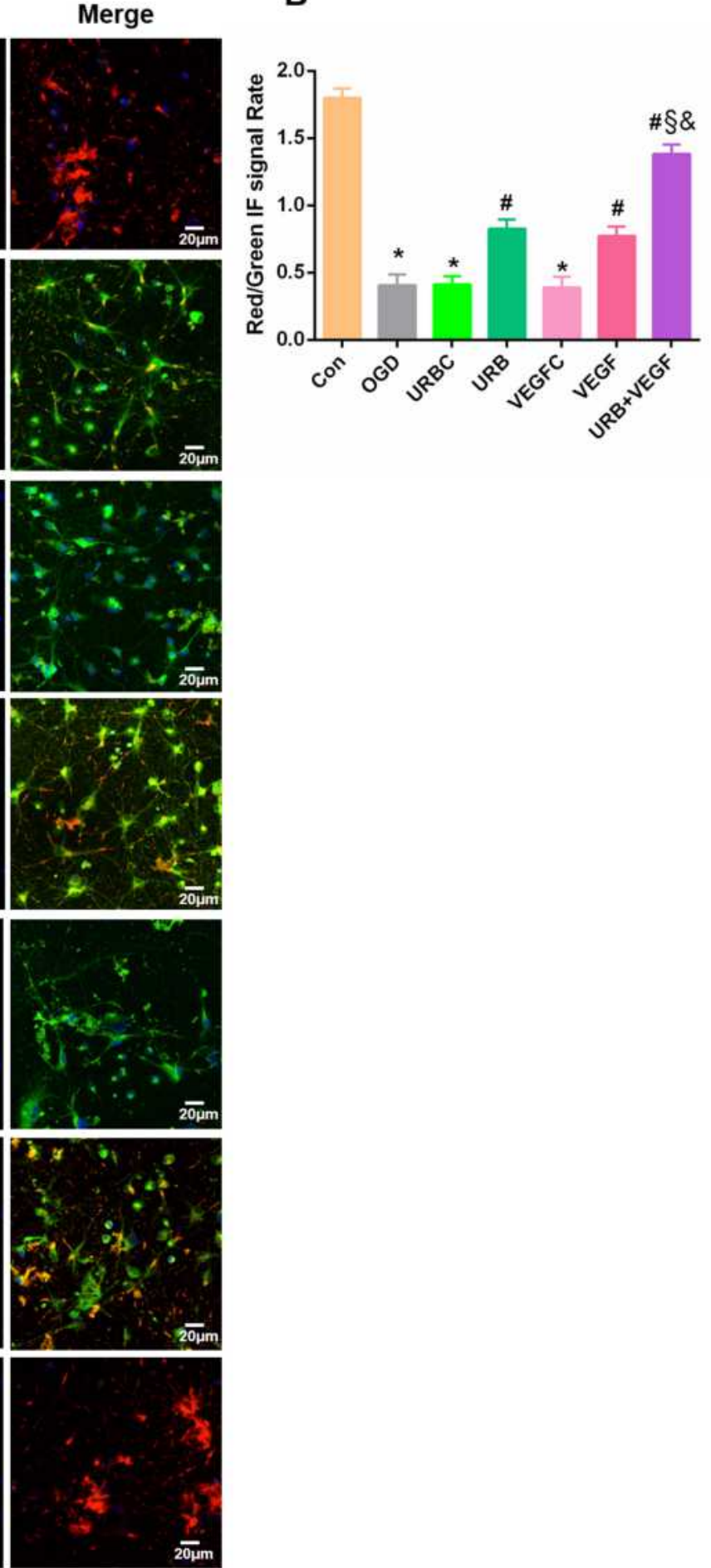

Figure 6 Effects of VEGF-A NF membrane and URB597 on mitochondrial membrane potential (MMP). (A) Representative immunofluorescence of mitochondrial JC-I in neurons. (B) MMP as estimated by JC-I red/green immunofluorescence signal rate. ${ }^{*} \mathrm{P}<0.05$ vs Con; ${ }^{\#} \mathrm{P}<0.05$ vs $\mathrm{OGD}$; ${ }^{\S} \mathrm{P}<0.05$ vs URB; ${ }^{\&} \mathrm{P}<0.05$ vs VEGF.

cellular survival, neurovascular unit remodeling, and apoptosis. ${ }^{32}$ Here, the levels of proinflammatory cytokines TNF- $\alpha$, IL-1 $\beta$, COX-2, and iNOS were measured by RTPCR (Figure 7C-F). Compared with the control group, the levels of all pro-inflammatory cytokines were raised in the OGD group, the URBC group, and the VEGFC group (all $\mathrm{p}<0.05$, vs Con group), while URB597 treatment during OGD significantly reduced expression levels (all $\mathrm{p}<0.05$, 

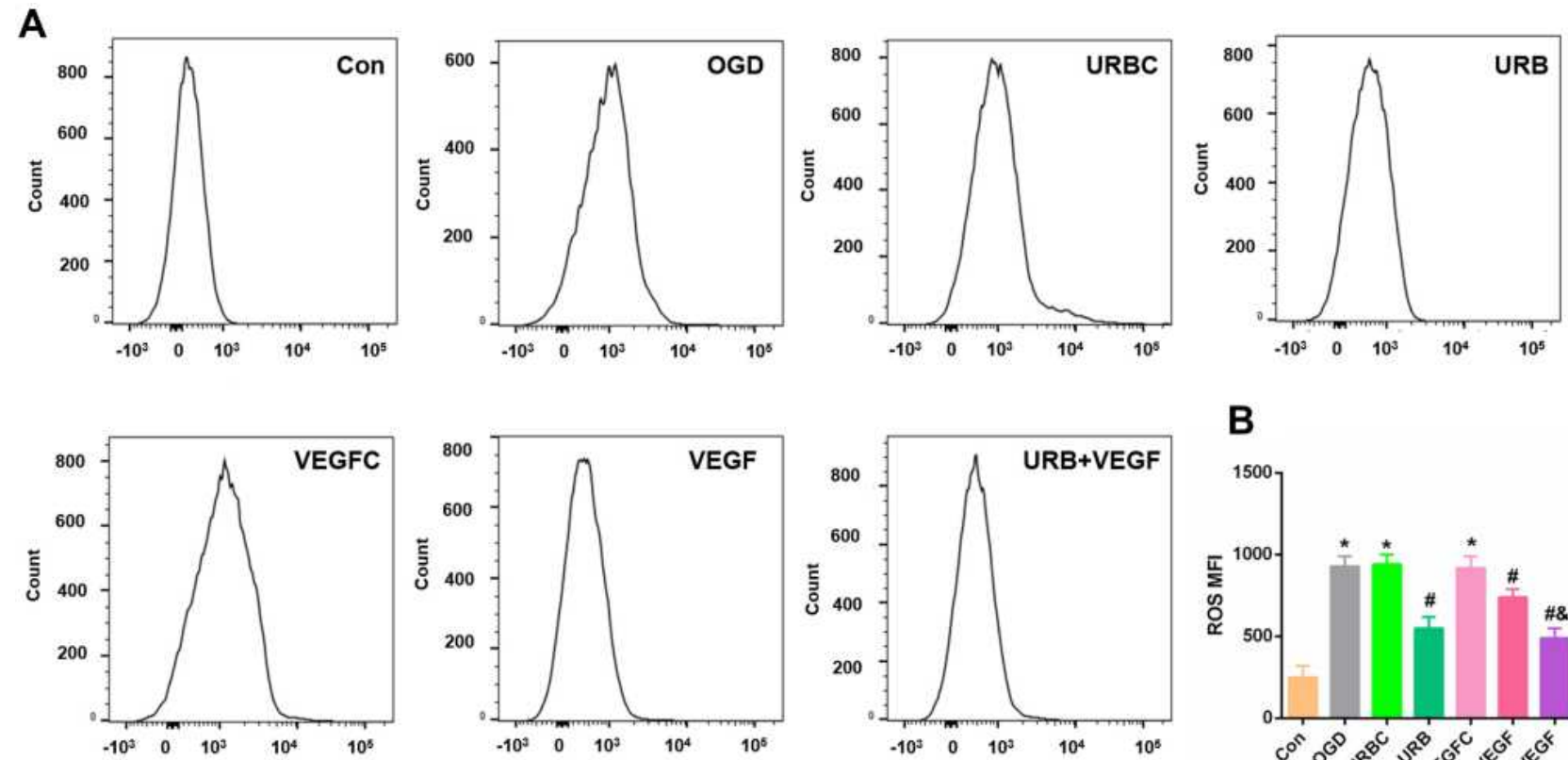

\section{B}
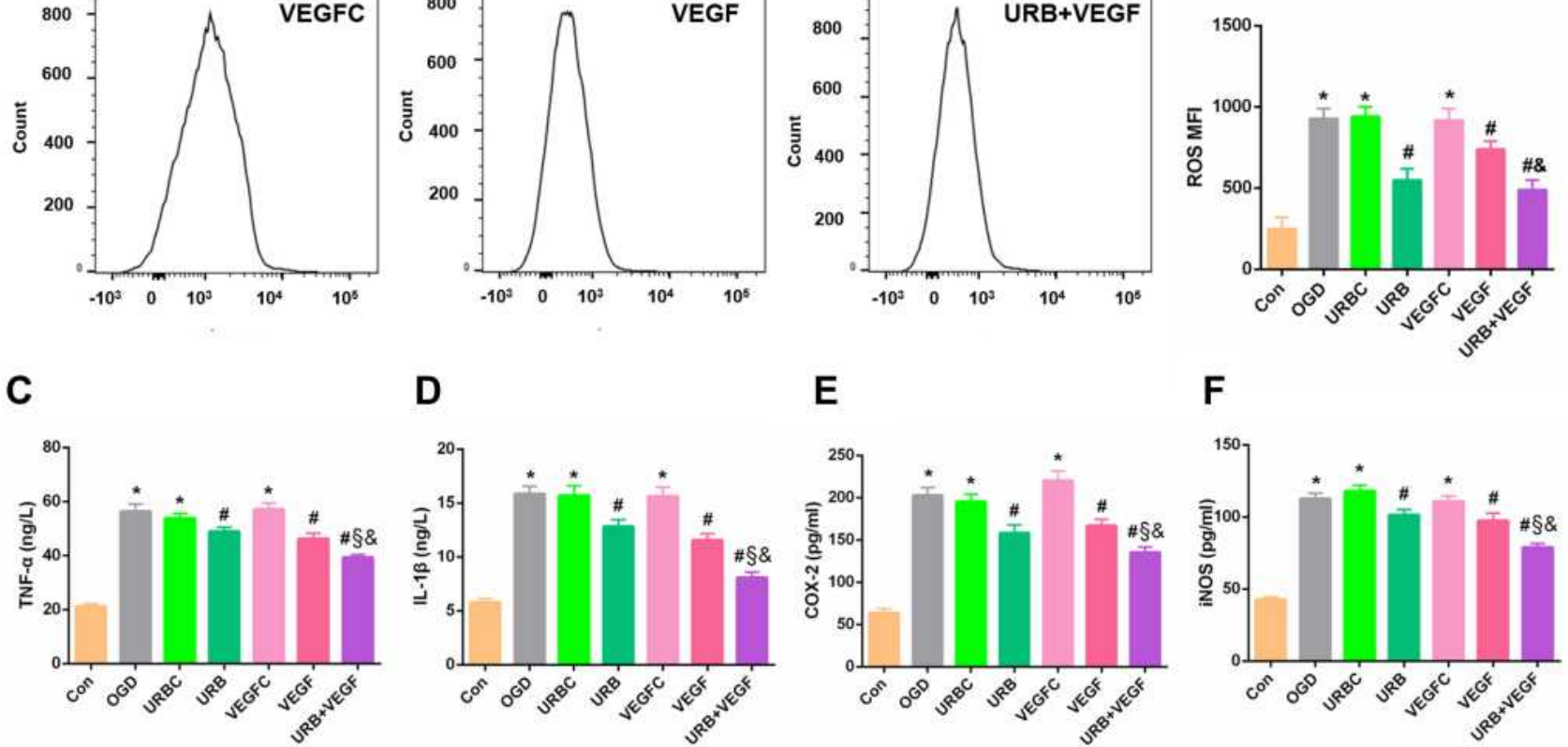

$\mathbf{F}$

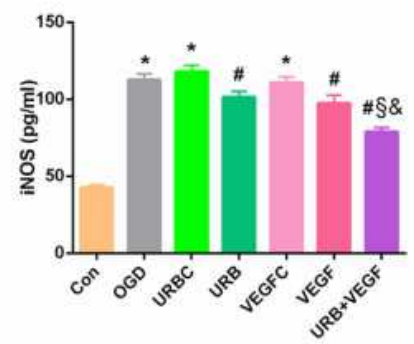

Figure 7 Effects of VEGF-A NF membrane and URB597 on mitochondria ROS and inflammatory responses. (A) Representative histograms of flow cytometric evaluation of the cell-associated DCF-DA. (B) Quantitative analysis of cellular ROS levels. (C-F) Quantitative analysis of pro-inflammatory factors, such as TNF- $\alpha$, IL-I $\beta$, COX-2, and iNOS. *P $<0.05$ vs Con; ${ }^{\#} \mathrm{P}<0.05$ vs OGD; ${ }^{\circledR} \mathrm{P}<0.05$ vs URB; ${ }^{\&} \mathrm{P}<0.05$ vs VEGF.

vs OGD group) as did VEGF-A NF membrane treatment (all $\mathrm{p}<0.05$, vs OGD group). Furthermore, compared with the URB group or compared with the VEGF group, the levels of proinflammatory cytokine were lower in the URB + VEGF group ( $\mathrm{p}<0.05$, respectively), suggesting that dual treatment with URB597 and VEGF-A can best suppress the OGD-induced inflammatory response.

\section{VEGF-A NF Membrane and URB597 Treatments Activate CBIR/PI3K/AKT/ BDNF Signaling, Exerting Synergistic Neuroprotective Effects}

The PI3K/AKT signaling pathway is a major mediator of the transduction of multiple cellular signals, and its activation is reported to enhance the ischemia-induced neurogenesis and promote cell survival. $^{34}$ However, its relationships with upstream and downstream molecules are complex. We further detected the expression of CB1R/PI3K/AKT/BDNF signaling elements using Western blotting (Figure 8A). CB1R, p-PI3K, and p-AKT levels significantly decreased in the OGD group compared with the Con group (all $\mathrm{p}<0.05$ ) (Figure 8B-D). However, those changes were abrogated by chronic treatment with VEGF-A NF membrane and URB597 (all p < 0.05, vs OGD group) (Figure 8B-D). We observed no obvious differences in the protein levels among the OGD group, the URBC group, and the VEGFC group. Co-treatment with URB597 and VEGF-A NF membrane increased the protein levels more substantially than treatment with URB or VEGF alone, exerting synergistic neuroprotective effects.

In addition, BDNF is vital for neuronal survival. The levels of BDNF and TrkB were dramatically decreased after OGD, but increased after treatment with URB597 and VEGF-A, which was accompanied by a reversal in 


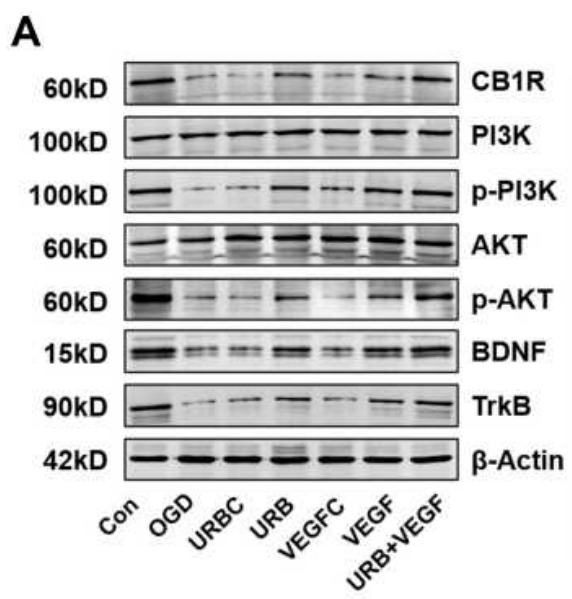

D

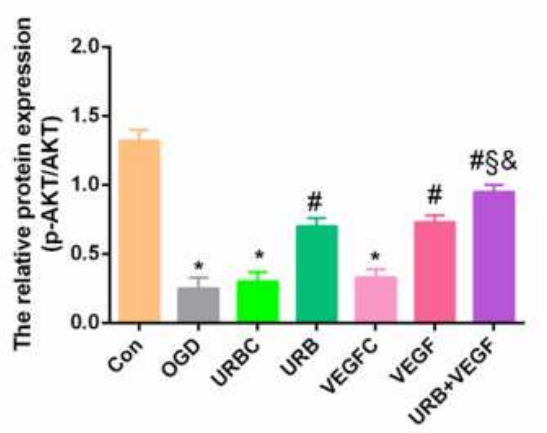

B

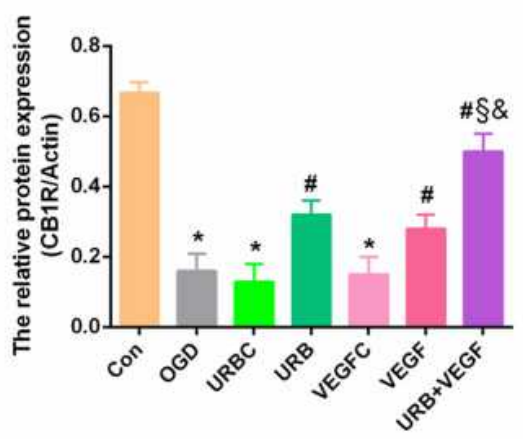

E

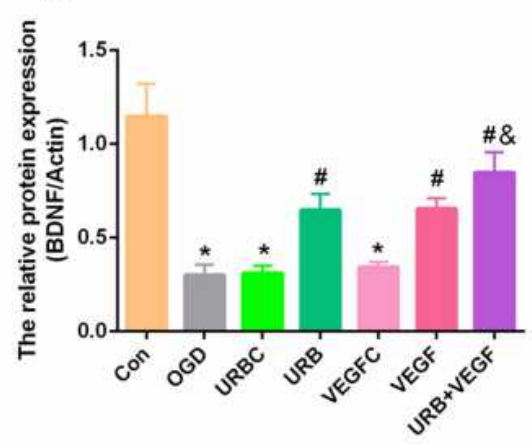

C

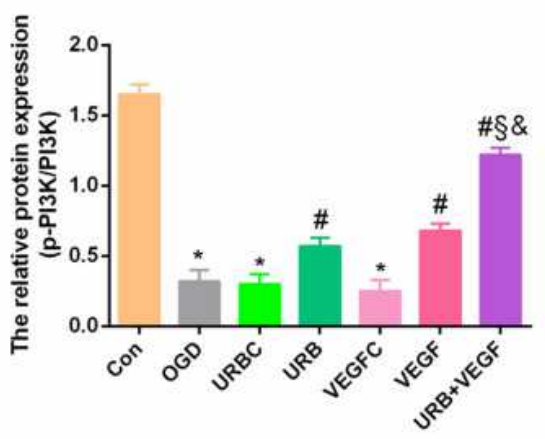

$\mathbf{F}$

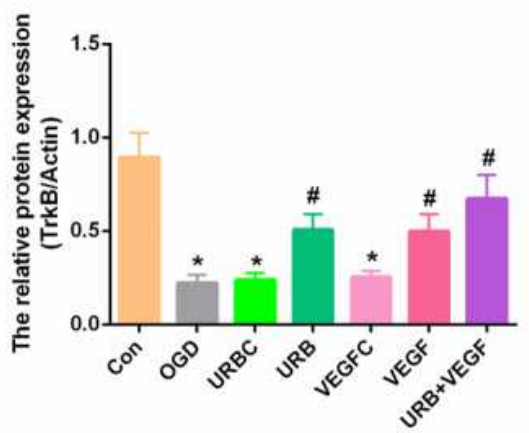

Figure 8 Effects of VEGF-A NF membrane and URB597 on CBIR signaling pathway components. (A) Representative Western blot of CBIR, PI3K, P-PI3K, AKT, p-AKT, BDNF, and TrkB. (B-F) Quantitative analysis of the expressions of proteins. ${ }^{* P}<0.05$ vs Con; ${ }^{\#} \mathrm{P}<0.05$ vs OGD; ${ }^{\S} \mathrm{P}<0.05$ vs URB; ${ }^{\circledR} \mathrm{P}<0.05$ vs VEGF.

apoptosis (Figures $5 \mathrm{~A}$ and $8 \mathrm{~A}$ ). Quantitative analysis showed that the expressions of BDNF signaling elements in the VEGF group and the URB+VEGF group were also significantly increased (all, $\mathrm{p}<0.05$ ) (Figure $8 \mathrm{E}$ and $\mathrm{F}$ ). Collectively, these results suggest that the neuroprotective synergistic effects of VEGF-A NF membrane and URB597 involve activation of the CR1R/PI3K/AKT/ BDNF pathway.

\section{Discussion}

Usage of nanomedicine materials is rapidly expanding as they promise revolutionary advances in the diagnosis and treatment of cerebrovascular disorders including cerebral small vessel diseases, ischemia-reperfusion injury, and stroke. ${ }^{35}$ There are many methods for fabricating NFs, such as drawing, template synthesis and assembly, and electrospinning. ${ }^{36}$ Electrospinning is a superior technology that is able to produce continuous NFs by employing LBL self-assembly technology. The LBL self-assembling electrospun NF is a promising medium for the continuous and controlled delivery of active substances for neural injury repair and tissue engineering. ${ }^{29,37}$ Our team previously reported that a biomimetic scaffold was deposited on the surface of PCL NFs to enable the local delivery of proteins to diseased tissues. ${ }^{28}$ In this study, we successfully fabricated a nanofibrous of VEGF-A loaded PCL membrane by using LBL. This method of incorporating the growth factor within the layers allowed it to be slowly released. ELISA assay showed that VEGF-A was slowly released over more than one month with inherent biodegradability. Furthermore, as shown by SEM, the ultramicroscopic morphology of the PCL NFs changed with VEGF-A embedding, providing an appropriate 3D microenvironment for cell adhesion and growth. However, as the protein load and numbers of layers increased, the average diameter also increased in the different layers, which affected the biochemical functions, such as scaffold hydrophilicity and cytotoxicity. Some studies have suggested that thinner NFs exhibit increased cell adhesion. ${ }^{38,39}$ Therefore, we speculated that the NFs with 15 or 20 layers with large diameters would not be favorable for neuron growth.

VEGF-A is a homodimeric disulfide bound glycoprotein that primarily promotes endothelial growth, and it also has neurotrophic and neuroprotective effects on neuronal cells within brain ischemia. ${ }^{40}$ However, in acute or chronic ischemic 
cerebral insults, VEGF-A with its complex underlying mechanisms can be either beneficial or destructive. ${ }^{41}$ This double-edged sword effect is closely related to its level and phase. VEGF-A could help retain the membrane potential of pyramidal neurons in the early stage of OGD (20 min), indicating an acute neuroprotective effect on OGD-induced neuronal injury. ${ }^{42}$ In hypoxic or ischemic preconditioning, VEGF-A plays an important role in late-phase changes in modulating targets. $^{43}$ We previously found that VEGF-A mRNA and protein levels dynamically changed with significant increases over 24 h postoperatively, peaking by 7 days, and decreasing by 21 days in chronic cerebral ischemia, which was associated with angiogenesis, blood-brain barrier dysfunction, and neurovascular remodeling. ${ }^{44}$ In a stroke, acute administration of exogenous VEGF-A is detrimental. ${ }^{41}$ Therefore, it is worth investigating ways to appropriately control the level of VEGFA to make it beneficial. The NF materials embellished with VEGF-A makes this possible, which also exhibit beneficial effects and clinical translational prospects for treating diseases, such as creating excellent microenvironmental stimuli for ischemia-associated neuronal regeneration and vascular remodeling in traumatic brain injury or indirect extracranial-intracranial (EC-IC) vascular bypass procedures (ie, encephalomyosynangiosis). ${ }^{16,17,45,46}$

Mitochondria are critical cellular organelles that maintain energy and metabolism, stress response, and neuronal activities in the brain. Mitochondrial dysfunction is a contributing factor to the pathology of multiple human conditions including OGD. ${ }^{47}$ During OGD episodes, the neuron cannot utilize mitochondrial mechanisms that allow it to survive and maintain homeostasis, which evokes MMP disruption. MMP impairment is a marker of subsequent ischemic neuronal death because it further leads to mitochondrial oxidative stress and inflammatory responses, which make it even worse. ${ }^{48}$ Here, upregulation of ROS and inflammatory factors were found in the OGD group, indicating mitochondrial dysfunction trigger a series of cascades. VEGF-A and URB597 could enhance primary hippocampal neuron survival and decrease the apoptosis by protecting mitochondria. OGD-induced increments in mitochondrial ROS, TNF- $\alpha$, IL$1 \beta$, COX-2, and iNOS were inhibited by VEGF-A and URB597. VEGF-A and URB597 showed clear mitochondrial protective effects not only by preventing mitochondrial MMP disruption but also by reducing mitochondria ROS production and neuroinflammation, which is consistent with previous studies. $^{49,50}$

OGD results in ischemic neuronal damage through activation of autophagy, neuroinflammatory responses, and induction of apoptosis in the hippocampal neuron in vitro. ${ }^{51}$ OGD-induced apoptotic cell death occurs by the inhibiting the PI3K/AKT and BDNF/TrkB pathways, ${ }^{40,52}$ and the neuroprotective effects of both URB597 and VEGF-A may be dependent on the activities of these two pathways. We previously demonstrated that URB597 could improve chronic cerebral ischemia-induced neuronal degeneration and apoptosis via activating CB1R/ AKT signaling. ${ }^{25}$ ESC takes a significant role via several downstream signaling pathways in ischemic tolerance and protection from ischemic stroke. ${ }^{53} \mathrm{CB} 1 \mathrm{R}$ activation is one prime underlying mechanism, it can be mediated by URB597, as has been substantiated. ${ }^{54}$ URB597 increases CB1R-mediated neuronal survival and neurotransmission, meanwhile up-regulating the levels of BDNF expression in the brain. ${ }^{55,56}$ Recently, we found that brain ischemia reduced BDNF expression in the cerebral cortex and hippocampus in rats with vascular dementia, which was reversed by chronic URB597 treatment. ${ }^{57} \mathrm{CBR}$ has been shown to have protective effects on hippocampal neurons in brain ischemia involving the PI3K signaling cascades. ${ }^{58}$ On the other hand, VEGF-A protects neurons against transient middle cerebral artery occlusion or chronic cerebral ischemia by stimulating PI3K/AKT/mTOR signaling. ${ }^{59,60}$ In in vivo and in vitro ischemia, VEGF-A could ameliorate cognitive impairment and synaptic plasticity via improving neuronal viability and function through acting on the PI3K/AKT/VEGFR-2 pathway. ${ }^{42}$ Blocking VEGF-A results in inhibiting adult hippocampal neuron growth via the PI3K/AKT-dependent mechanism. ${ }^{61}$ In this study, we showed for the first time that the synergistic actions of VEGF-A and URB597 on OGD-induced apoptotic cell death are mediated by $\mathrm{CB} 1 \mathrm{R} / \mathrm{PI} 3 \mathrm{~K} / \mathrm{AKT} /$ BDNF activation. Co-administration of VEGF-A and URB597 increased CB1R expression, and enhanced $\mathrm{PI}$ K/AKT phosphorylation without affecting total PI3K and AKT levels. PI3K and AKT phosphorylation and subsequent activation can be prevented by the CB1R antagonist AM251. ${ }^{62}$ We previously reported that WIN55,212-2 (a nonselective CBR agonist) and URB597 could ameliorate hippocampal neuronal damage and cognitive dysfunction in rats with chronic cerebral ischemia, both of which were reversed by the CB1R antagonist AM281. ${ }^{63}$ In fact, accumulating evidence suggests that CB1R can increase not merely VEGF-A expression, ${ }^{64}$ but activate multiple pathways, including PI3K/AKT/ BDNF signaling in promoting striatal neuron survival. ${ }^{65}$ 
There are several limitations to this study. Firstly, we only investigated the effect and mechanisms of VEGF-A and URB597 in primarily cultured hippocampal neurons under OGD. Their effects on cerebral ischemia in animals as well as on neurological functions need to be further assessed, especially intervention using the CB1R antagonist. Secondly, there is a lack of cytotoxicity tests on the material itself. Cytotoxicity of NFs is generally examined according to ISO 10993-5, analyzing the biosafety of medical materials or devices on living organisms. However, previous experiments have shown that PCL NF alone does not affect cell growth. ${ }^{66,67}$ In addition, the relatively short experimental period provided insufficient evidence to support the long-term solubility of the VEGFA loaded NF membrane. The degradation of PCL scaffolds is very slow, which remains a challenge. Fortunately, raising the temperature and the ratio of PEG to PCL can lead to significant acceleration of the degradation of PCL. ${ }^{68}$

\section{Conclusion}

Generally, these data are of clinical translational significance for the treatment of ischemic neuronal injuries

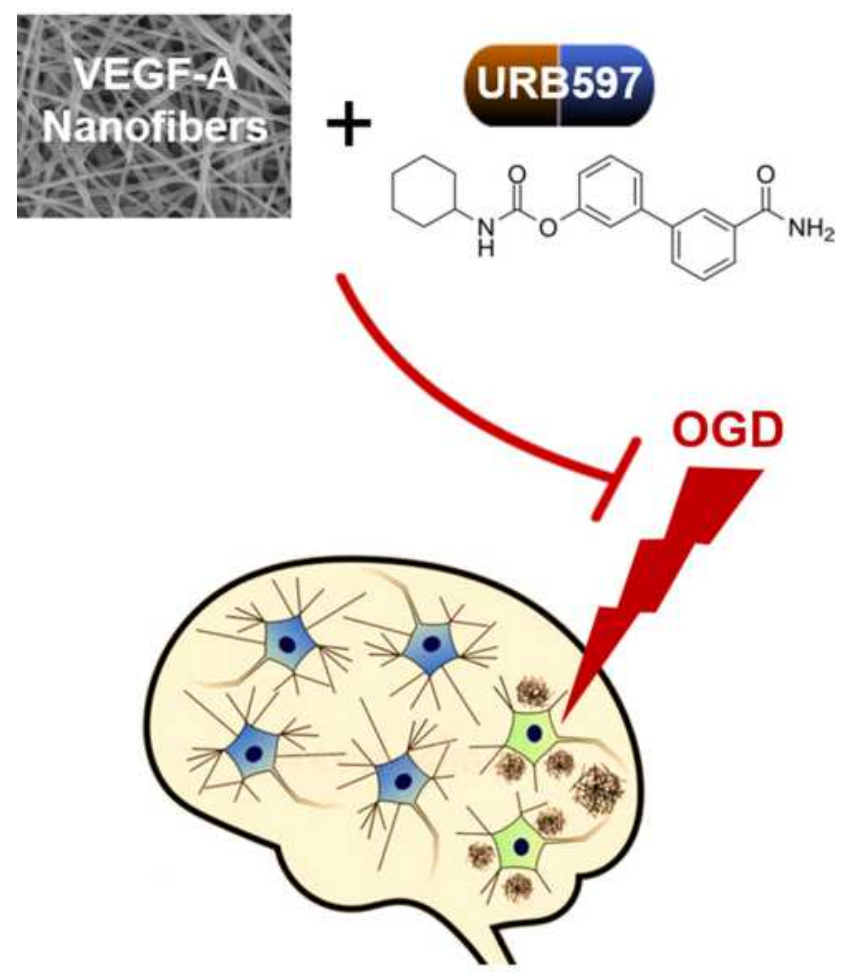

\section{Brain ischemic neuronal injury}

Figure 9 The VEGF-A NF membrane and URB597 show positive synergistic neuroprotective effects against OGD-induced brain ischemic neuronal injury, exhibiting a therapeutic value in ischemic cerebrovascular diseases.
(Figure 9). The mitochondrial oxidative stress cascades and $\mathrm{CB} 1 \mathrm{R} / \mathrm{PI} 3 \mathrm{~K} / \mathrm{AKT} / \mathrm{BDNF}$ pathway play important roles in the pathogenesis of OGD-induced neuronal damage. VEGF-A released by the NF membrane and URB597 showed positive synergistic neuroprotective effects by inhibiting oxidative stress and activating CB1R signaling, suggesting that VEGF-A loaded NF membranes and the FAAH inhibitor URB597 may be of therapeutic value in ischemic cerebrovascular diseases.

\section{Ethical Statement}

Experiments were performed under a project license (NO. TJ20191012-A006) granted by institutional ethics board of Tongji University School of Medicine, in compliance with the National institutional guidelines for the care and use of animals.

\section{Acknowledgments}

The authors thank Dr. Hai Fei from the Institute for Biomedical Engineering and Nanoscience in Shanghai East Hospital of Tongji University for assistance and support for the electrospinning technique. The study was supported by the National Nature Science Foundation of China (Grant Nos. 81771410, 81974207, 82001383), Program of Outstanding Young Scientists of Tongji Hospital of Tongji University (HBRC2008) and the Clinical Research Project of Shanghai Municipal Health Commission (20204Y0074).

\section{Disclosure}

The authors report no conflicts of interest in this work.

\section{References}

1. Mehanna R, Jankovic J. Movement disorders in cerebrovascular disease. Lancet Neurol. 2013;12(6):597-608. doi:10.1016/S14744422(13)70057-7.

2. Barthels D, Das H. Current advances in ischemic stroke research and therapies. Biochim Biophys Acta Mol Basis Dis. 2020;1866(4):165260. doi:10.1016/j.bbadis.2018.09.012.

3. Ryou MG, Mallet RT. An in vitro oxygen-glucose deprivation model for studying ischemia-reperfusion injury of neuronal cells. Methods Mol Biol. 2018;1717:229-235. doi:10.1007/978-1-4939-7526-6 18.

4. Tasca CI, Dal-Cim T, Cimarosti H. In vitro oxygen-glucose deprivation to study ischemic cell death. Methods Mol Biol. 2015;1254:197-210. doi:10.1007/978-1-4939-2152-2 15.

5. Eatemadi A, Daraee H, Zarghami N, et al. Nanofiber: synthesis and biomedical applications. Artif Cells Nanomed Biotechnol. 2016;44 (1):111-121. doi:10.3109/21691401.2014.922568.

6. Rasouli R, Barhoum A, Bechelany M, et al. Nanofibers for biomedical and healthcare applications. Macromol Biosci. 2019;19(2):e1800256. doi:10.1002/mabi.201800256.

7. Bonnard T, Gauberti M, Martinez de Lizarrondo S, et al. Recent advances in nanomedicine for ischemic and hemorrhagic stroke. Stroke. 2019;50 (5):1318-1324. doi:10.1161/STROKEAHA.118.022744 
8. Chu J, Chen L, Mo Z, et al. An atorvastatin calcium and poly (L-lactide-co-caprolactone) core-shell nanofiber-covered stent to treat aneurysms and promote reendothelialization. Acta Biomater. 2020;111:102-117. doi:10.1016/j.actbio.2020.04.044.

9. Wang TY, Forsythe JS, Nisbet DR, et al. Promoting engraftment of transplanted neural stem cells/progenitors using biofunctionalised electrospun scaffolds. Biomaterials. 2012;33(36):9188-9197. doi:10.1016/j.biomaterials.2012.09.013.

10. Chen S, Li R, Li X, et al. Electrospinning: an enabling nanotechnology platform for drug delivery and regenerative medicine. Adv Drug Deliv Rev. 2018;132:188-213. doi:10.1016/j.addr.2018.05.001.

11. Wang TW, Chang KC, Chen LH, et al. Effects of an injectable functionalized self-assembling nanopeptide hydrogel on angiogenesis and neurogenesis for regeneration of the central nervous system. Nanoscale. 2017;9(42):16281-16292. doi:10.1039/c7nr06528k.

12. Apte RS, Chen DS, Ferrara N. VEGF in signaling and disease: beyond discovery and development. Cell. 2019;176(6):1248-1264. doi:10.1016/j.cell.2019.01.021.

13. Arany Z, Foo SY, Ma Y, et al. HIF-independent regulation of VEGF and angiogenesis by the transcriptional coactivator PGC-1alpha. Nature. 2008;451(7181):1008-1012. doi:10.1038/nature06613.

14. Liu D, Ye Y, Xu L, et al. Icariin and mesenchymal stem cells synergistically promote angiogenesis and neurogenesis after cerebral ischemia via PI3K and ERK1/2 pathways. Biomed Pharmacother. 2018;108:663-669. doi:10.1016/j.biopha.2018.09.071.

15. Matsusaki M, Sakaguchi H, Serizawa T, et al. Controlled release of vascular endothelial growth factor from alginate hydrogels nano-coated with polyelectrolyte multilayer films. J Biomater Sci Polym Ed. 2007;18(6):775-783. doi:10.1163/156856207781034160.

16. Hecht N, Marushima A, Nieminen M, et al. Myoblast-mediated gene therapy improves functional collateralization in chronic cerebral hypoperfusion. Stroke. 2015;46(1):203-211. doi:10.1161/ STROKEAHA.114.006712.

17. Marushima A, Nieminen M, Kremenetskaia I, et al. Balanced single-vector co-delivery of VEGF/PDGF-BB improves functional collateralization in chronic cerebral ischemia. J Cereb Blood Flow Metab. 2020;40(2):404-419. doi:10.1177/0271678X18818298.

18. Liu SM, Xiao ZF, Li X, et al. Vascular endothelial growth factor activates neural stem cells through epidermal growth factor receptor signal after spinal cord injury. CNS Neurosci Ther. 2019;25 (3):375-385. doi:10.1111/cns.13056.

19. Abdel-Latif RG, Rifaai RA, Amin EF. Empagliflozin alleviates neuronal apoptosis induced by cerebral ischemia/reperfusion injury through HIF-1alpha/VEGF signaling pathway. Arch Pharm Res. 2020;43(5):514-525. doi:10.1007/s12272-020-01237-y.

20. Lin R, Cai J, Kenyon L, et al. Systemic factors trigger vasculature cells to drive notch signaling and neurogenesis in neural stem cells in the adult brain. Stem Cells. 2019;37(3):395-406. doi:10.1002/ stem. 2947.

21. Cristino L, Bisogno T, Di Marzo V. Cannabinoids and the expanded endocannabinoid system in neurological disorders. Nat Rev Neurol. 2020;16(1):9-29. doi:10.1038/s41582-019-0284-z.

22. D'Souza DC, Cortes-Briones J, Creatura G, et al. Efficacy and safety of a fatty acid amide hydrolase inhibitor (PF-04457845) in the treatment of cannabis withdrawal and dependence in men: a double-blind, placebo-controlled, parallel group, phase 2a single-site randomised controlled trial. Lancet Psychiatry. 2019;6(1):35-45. doi:10.1016/ S2215-0366(18)30427-9.

23. Kerbrat A, Ferre JC, Fillatre P, et al. Acute neurologic disorder from an inhibitor of fatty acid amide hydrolase. $N$ Engl J Med. 2016;375 (18):1717-1725. doi:10.1056/NEJMoa1604221.

24. Degn M, Lambertsen KL, Petersen G, et al. Changes in brain levels of $\mathrm{N}$-acylethanolamines and 2-arachidonoylglycerol in focal cerebral ischemia in mice. $J$ Neurochem. 2007;103(5):1907-1916. doi:10.1111/j.1471-4159.2007.04892.x.
25. Wang D, Lin Q, Su S, et al. URB597 improves cognitive impairment induced by chronic cerebral hypoperfusion by inhibiting mTOR-dependent autophagy. Neuroscience. 2017;344:293-304. doi:10.1016/j.neuroscience.2016.12.034.

26. Su SH, Wu YF, Wang DP, et al. Inhibition of excessive autophagy and mitophagy mediates neuroprotective effects of URB597 against chronic cerebral hypoperfusion. Cell Death Dis. 2018;9(7):733. doi:10.1038/s41419-018-0755-y

27. Han F, Zhang P, Sun Y, et al. Hydroxyapatite-doped polycaprolactone nanofiber membrane improves tendon-bone interface healing for anterior cruciate ligament reconstruction. Int $J$ Nanomedicine. 2015;10:7333-7343. doi:10.2147/IJN.S92099.

28. Han F, Zhang P, Chen T, et al. A LbL-assembled bioactive coating modified nanofibrous membrane for rapid tendon-bone healing in ACL reconstruction. Int J Nanomedicine. 2019;14:9159-9172. doi:10.2147/IJN.S214359.

29. Zhang P, Han F, Chen T, et al. "Swiss roll"-like bioactive hybrid scaffolds for promoting bone tissue ingrowth and tendon-bone healing after anterior cruciate ligament reconstruction. Biomater Sci. 2020;8(3):871-883. doi:10.1039/c9bm01703h.

30. Roppongi RT, Champagne-Jorgensen KP, Siddiqui TJ. Low-density primary hippocampal neuron culture. J Vis Exp. 2017;(122). doi: $10.3791 / 55000$.

31. Shi L, Zhang J, Wang Y, et al. Sirt1 regulates oxidative stress in oxygen-glucose deprived hippocampal neurons. Front Pediatr. 2020;8:455. doi:10.3389/fped.2020.00455.

32. Wang DP, Yin H, Kang K, et al. The potential protective effects of cannabinoid receptor agonist WIN55,212-2 on cognitive dysfunction is associated with the suppression of autophagy and inflammation in an experimental model of vascular dementia. Psychiatry Res. 2018;267:281-288. doi:10.1016/j.psychres.2018.06.012.

33. Wei P, Yang F, Zheng Q, et al. The potential role of the NLRP3 inflammasome activation as a link between mitochondria ROS generation and neuroinflammation in postoperative cognitive dysfunction. Front Cell Neurosci. 2019;13:73. doi:10.3389/fncel.2019.00073.

34. Kilic U, Caglayan AB, Beker MC, et al. Particular phosphorylation of PI3K/Akt on Thr308 via PDK-1 and PTEN mediates melatonin's neuroprotective activity after focal cerebral ischemia in mice. Redox Biol. 2017;12:657-665. doi:10.1016/j.redox.2017.04.006.

35. Gilmore JL, Yi X, Quan L, et al. Novel nanomaterials for clinical neuroscience. J Neuroimmune Pharmacol. 2008;3(2):83-94. doi:10.1007/s11481-007-9099-6.

36. Maduraiveeran G, Sasidharan M, Ganesan V. Electrochemical sensor and biosensor platforms based on advanced nanomaterials for biological and biomedical applications. Biosens Bioelectron. 2018;103:113-129. doi:10.1016/j.bios.2017.12.031.

37. Zhang K, Chooi WH, Liu S, et al. Localized delivery of CRISPR/ dCas9 via layer-by-layer self-assembling peptide coating on nanofibers for neural tissue engineering. Biomaterials. 2020;256:120225. doi:10.1016/j.biomaterials.2020.120225.

38. Park JK, Pham-Nguyen OV, Yoo HS. Coaxial electrospun nanofibers with different shell contents to control cell adhesion and viability. ACS Omega. 2020;5(43):28178-28185. doi:10.1021/acsomega.0c03902.

39. Kadler K. Matrix loading: assembly of extracellular matrix collagen fibrils during embryogenesis. Birth Defects Res C Embryo Today. 2004;72(1):1-11. doi:10.1002/bdrc.20002.

40. Choi NY, Kim JY, Hwang M, et al. Atorvastatin rejuvenates neural stem cells injured by oxygen-glucose deprivation and induces neuronal differentiation through activating the PI3K/Akt and ERK pathways. Mol Neurobiol. 2019;56(4):2964-2977. doi:10.1007/s12035-018-1267-6.

41. Geiseler SJ, Morland C. The janus face of VEGF in stroke. Int J Mol Sci. 2018;19(5):1362. doi:10.3390/ijms19051362.

42. Yang J, Yao Y, Chen T, et al. VEGF ameliorates cognitive impairment in in vivo and in vitro ischemia via improving neuronal viability and function. Neuromolecular Med. 2014;16(2):376-388. doi:10.1007/s12017-013-8284-4. 
43. Li S, Hafeez A, Noorulla F, et al. Preconditioning in neuroprotection: from hypoxia to ischemia. Prog Neurobiol. 2017;157:79-91. doi:10.1016/j.pneurobio.2017.01.001.

44. Hai J, Li ST, Lin Q, et al. Vascular endothelial growth factor expression and angiogenesis induced by chronic cerebral hypoperfusion in rat brain. Neurosurgery. 2003;53(4):963-970; discussion 970-962 doi:10.1227/01.neu.0000083594.10117.7a.

45. Li G, Han Q, Lu P, et al. Construction of dual-biofunctionalized chitosan/collagen scaffolds for simultaneous neovascularization and nerve regeneration. Research. 2020;2020:2603048. doi:10.34133/ 2020/2603048

46. Zhang J, Liu X, Ma K, et al. Collagen/heparin scaffold combined with vascular endothelial growth factor promotes the repair of neurological function in rats with traumatic brain injury. Biomater Sci. 2020. doi:10.1039/c9bm01446b.

47. Flannery PJ, Trushina E. Mitochondrial dynamics and transport in Alzheimer's disease. Mol Cell Neurosci. 2019;98:109-120. doi:10.1016/j.mcn.2019.06.009.

48. Iijima T. Mitochondrial membrane potential and ischemic neuronal death. Neurosci Res. 2006;55(3):234-243. doi:10.1016/j. neures.2006.04.005.

49. Keilhoff G, Schild L, Fansa H. Minocycline protects Schwann cells from ischemia-like injury and promotes axonal outgrowth in bioartificial nerve grafts lacking Wallerian degeneration. Exp Neurol. 2008;212(1):189-200. doi:10.1016/j.expneurol.2008.03.028.

50. Shan Y, Tan S, Lin Y, et al. The glucagon-like peptide-1 receptor agonist reduces inflammation and blood-brain barrier breakdown in an astrocyte-dependent manner in experimental stroke. $J$ Neuroinflammation. 2019;16(1):242． doi:10.1186/s12974-0191638-6

51. Zhi J, Duan B, Pei J, et al. Daphnetin protects hippocampal neurons from oxygen-glucose deprivation-induced injury. $J$ Cell Biochem. 2019;120(3):4132-4139. doi:10.1002/jcb.27698.

52. Du L, Mei Z, Huang Y, et al. Protection of the Geum japonicum Thunb. var. chinense extracts against oxygen-glucose deprivation and re-oxygenation induced astrocytes injury via BDNF/PI3K/Akt/CREB pathway. Biomed Pharmacother. 2020;127:110123. doi:10.1016/j. biopha.2020.110123.

53. Zhuang Q, Dai C, Yang L, et al. Stimulated CB1 cannabinoid receptor inducing ischemic tolerance and protecting neuron from cerebral ischemia. Cent Nerv Syst Agents Med Chem. 2017;17(2):141-150. doi:10.2174/1871524916666160504104624

54. Chavira-Ramos K, Orozco-Morales M, Karasu C, et al. URB597 prevents the short-term excitotoxic cell damage in rat cortical slices: role of cannabinoid 1 receptors. Neurotox Res. 2020;39(2):146-155. doi:10.1007/s12640-020-00301-1.

55. Burstein O, Shoshan N, Doron R, et al. Cannabinoids prevent depressive-like symptoms and alterations in BDNF expression in a rat model of PTSD. Prog Neuropsychopharmacol Biol Psychiatry. 2018;84(Pt A):129-139. doi:10.1016/j.pnpbp.2018.01.026.

56. Bosier B, Muccioli GG, Lambert DM. The FAAH inhibitor URB597 efficiently reduces tyrosine hydroxylase expression through $\mathrm{CB}(1)$ and FAAH-independent mechanisms. Br J Pharmacol. 2013;169 (4):794-807. doi:10.1111/j.1476-5381.2012.02208.x.

International Journal of Nanomedicine

\section{Publish your work in this journal}

The International Journal of Nanomedicine is an international, peerreviewed journal focusing on the application of nanotechnology in diagnostics, therapeutics, and drug delivery systems throughout the biomedical field. This journal is indexed on PubMed Central, MedLine, CAS, SciSearch ${ }^{\mathbb{B}}$, Current Contents ${ }^{\mathbb{B}} /$ Clinical Medicine, $^{2}$
57. Wang DP, Lin Q, Kang K, et al. Preservation of spatial memory and neuroprotection by the fatty acid amide hydrolase inhibitor URB597 in a rat model of vascular dementia. Ann Transl Med. 2021;9(3):228. doi: $10.21037 /$ atm-20-4431

58. Palomba L, Motta A, Imperatore R, et al. Role of 2-arachidonoylglycerol and CB1 receptors in orexin-A-mediated prevention of oxygen-glucose deprivation-induced neuronal injury. Cells. 2020;9 (6):1507. doi:10.3390/cells9061507.

59. Wang L, Wang F, Liu S, et al. VEGF attenuates 2-VO induced cognitive impairment and neuronal injury associated with the activation of PI3K/Akt and Notch1 pathway. Exp Gerontol. 2018;102:93-100. doi:10.1016/j.exger.2017.12.010.

60. Perez-Alvarez MJ, Villa Gonzalez M, Benito-Cuesta I, et al. Role of mTORC1 controlling proteostasis after brain ischemia. Front Neurosci. 2018;12:60. doi:10.3389/fnins.2018.00060.

61. Fournier NM, Lee B, Banasr M, et al. Vascular endothelial growth factor regulates adult hippocampal cell proliferation through MEK/ ERK- and PI3K/Akt-dependent signaling. Neuropharmacology. 2012;63(4):642-652. doi:10.1016/j.neuropharm.2012.04.033

62. Ibrahim BM, Abdel-Rahman AA. Differential modulation of brainstem phosphatidylinositol 3-kinase/Akt and extracellular signal-regulated kinase 1/2 signaling underlies WIN55,212-2 centrally mediated pressor response in conscious rats. J Pharmacol Exp Ther. 2012;340(1):11-18. doi:10.1124/jpet.111.186858

63. Su SH, Wang YQ, Wu YF, et al. Cannabinoid receptor agonist WIN55,212-2 and fatty acid amide hydrolase inhibitor URB597 may protect against cognitive impairment in rats of chronic cerebral hypoperfusion via PI3K/AKT signaling. Behav Brain Res. 2016;313:334-344. doi:10.1016/j.bbr.2016.07.009

64. Lojpur T, Easton Z, Raez-Villanueva S, et al. Delta9Tetrahydrocannabinol leads to endoplasmic reticulum stress and mitochondrial dysfunction in human BeWo trophoblasts. Reprod Toxicol. 2019;87:21-31. doi:10.1016/j.reprotox.2019.04.008

65. Blazquez C, Chiarlone A, Bellocchio L, et al. The $\mathrm{CB}(1)$ cannabinoid receptor signals striatal neuroprotection via a PI3K/Akt/mTORC1/ BDNF pathway. Cell Death Differ. 2015;22(10):1618-1629. doi:10.1038/cdd.2015.11

66. Chen $\mathrm{CH}$, Chen SH, Shalumon KT, et al. Dual functional core-sheath electrospun hyaluronic acid/polycaprolactone nanofibrous membranes embedded with silver nanoparticles for prevention of peritendinous adhesion. Acta Biomater. 2015;26:225-235. doi:10.1016/j. actbio.2015.07.041

67. Jafari A, Amirsadeghi A, Hassanajili S, et al. Bioactive antibacterial bilayer PCL/gelatin nanofibrous scaffold promotes full-thickness wound healing. Int $J$ Pharm. 2020;583:119413. doi:10.1016/j. ijpharm.2020.119413

68. Felfel RM, Poocza L, Gimeno-Fabra M, et al. In vitro degradation and mechanical properties of PLA-PCL copolymer unit cell scaffolds generated by two-photon polymerization. Biomed Mater. 2016;11 (1):015011. doi:10.1088/1748-6041/11/1/015011
Journal Citation Reports/Science Edition, EMBase, Scopus and the Elsevier Bibliographic databases. The manuscript management system is completely online and includes a very quick and fair peer-review system, which is all easy to use. Visit http://www.dovepress.com/ testimonials.php to read real quotes from published authors. 